\title{
Basigin Associates with Integrin in Order to Regulate Perineurial Glia and Drosophila Nervous System Morphology
}

\author{
Amelia C. Hunter, Lindsay M. Petley-Ragan, ${ }^{\circledR}$ Mriga Das, and ${ }^{\circledR}$ Vanessa J. Auld \\ Department of Zoology, University of British Columbia, Vancouver, British Columbia V6T 1Z4, Canada
}

The Drosophila nervous system is ensheathed by a layer of outer glial cells, the perineurial glia, and a specialized extracellular matrix, the neural lamella. The function of perineurial glial cells and how they interact with the extracellular matrix are just beginning to be elucidated. Integrin-based focal adhesion complexes link the glial membrane to the extracellular matrix, but little is known about integrin's regulators in the glia. The transmembrane Ig domain protein Basigin/CD147/ EMMPRIN is highly expressed in the perineurial glia surrounding the Drosophila larval nervous system. Here we show that Basigin associates with integrin at the focal adhesions to uphold the structure of the glia-extracellular matrix sheath. Knockdown of Basigin in perineurial glia using RNAi results in significant shortening of the ventral nerve cord, compression of the glia and extracellular matrix in the peripheral nerves, and reduction in larval locomotion. We determined that Basigin is expressed in close proximity to integrin at the glial membrane, and that expression of the extracellular integrinbinding domain of Basigin is sufficient to rescue peripheral glial compression. We also found that a reduction in expression of integrin at the membrane rescues the ventral nerve cord shortening, peripheral glial compression, and locomotor phenotypes, and that reduction in the integrin-binding protein Talin can partially rescue glial compression. These results identify Basigin as a potential negative regulator of integrin in the glia, supporting proper glial and extracellular matrix ensheathment of the nervous system.

Key words: Integrin; extracellular matrix; glia; Drosophila; Neuroplatstin; EMMPRIN

\section{Significance Statement}

The glial cells and extracellular matrix play important roles in supporting and protecting the nervous system, but the interactions between these components have not been well characterized. Our study identified expression of a conserved Ig superfamily protein, Basigin, at the glial membrane of Drosophila where it associates with the integrin-based focal adhesion complexes to ensure proper ensheathment of the CNS and PNS. Loss of Basigin in the glia results in an overall compression of the nervous system due to integrin dysregulation, which causes locomotor defects in the animals. This underlies the importance of glia-matrix communication for structural and functional support of the nervous system.

Received June 6, 2019; revised Mar. 3, 2020; accepted Mar. 9, 2020

Author contributions: A.C.H., L.M.P.-R., and V.J.A. designed research; A.C.H., L.M.P.-R., and M.D. performed research; A.C.H., L.M.P.-R., M.D., and V.J.A. analyzed data; A.C.H., L.M.P.-R., and V.J.A. edited the paper; A.C.H., L.M.P.-R., and V.J.A. wrote the paper; L.M.P.-R. wrote the first draft of the paper.

The authors declare no competing financial interests.

This work was supported by Canadian Institute of Health Research and the Natural Sciences and Engineering Research Council. The Developmental Studies Hybridoma Bank, created by the National Institute of Child Health and Human Development of the National Institutes of Health and maintained at the University of lowa (Department of Biology, lowa City, IA 52242), provided monoclonal antibodies used in this study. Stocks obtained from the Bloomington Drosophila Stock Center (National Institutes of Health P400D018537) were used in this study. We thank Drs. Anne Ephrussi, Ken Prehoda, Bruce Reed, and Guy Tanentzapf for generously sharing fly lines and antibodies; and Drs. Tim O'Connor and Mary Gilbert for constructive comments on the manuscript.

Correspondence should be addressed to Vanessa J. Auld at auld@zoology.ubc.ca.

https://doi.org/10.1523/JNEUROSCI.1397-19.2020

Copyright $\odot 2020$ the authors

\section{Introduction}

In the nervous system, glial cells provide structural support and protection to the underlying axons, and express receptors to bind the overlying extracellular matrix (ECM). The ECM regulates the size and shape of the nervous system during development, and is essential for its structural integrity (Pastor-Pareja and $\mathrm{Xu}, 2011$ ). In Drosophila, ECM degradation by overexpression of matrix metalloproteinases causes deformation of the brain lobes and ventral nerve cord (VNC), and prevents proper glial sheath formation (Xie and Auld, 2011; Meyer et al., 2014). Similarly, in vertebrate neurons, the ECM plays an essential role in myelination of axons by the glia (Colognato and Tzvetanova, 2011).

Glia-ECM interactions are mediated by the integrin heterodimer in many glial cells. The extracellular heads of the $\alpha$ and $\beta$ 
integrin subunits bind ECM components, whereas the intracellular domain binds a host of adaptor proteins, including the actinbinding protein Talin, forming the focal adhesion complex (Sun et al., 2016). In Drosophila, glial knockdown of $\beta$-integrin expression causes CNS deformation and incomplete glial wrapping, similar to ECM degradation phenotypes (Xie and Auld, 2011; Meyer et al., 2014). Loss of $\beta$-integrin in mice also causes incomplete myelination of neurons (Feltri et al., 2002; Barros et al., 2003).

Intracellular binding and activation of integrin ("inside-out" signaling) promote ligand binding and increased cell adhesion (O’Toole et al., 1991; Calderwood et al., 1999; Hu and Luo, 2013). Extracellular binding of integrin to ligands results in activation of intracellular signaling ("outside-in" signaling), resulting in changes to cell shape, growth, and migration (Hu and Luo, 2013). The integrin dimer undergoes large-scale conformational changes based on its activation state: the dimer is bent while inactive, extended while activated; and the transmembrane portions of the $\alpha$ and $\beta$ subunits are separated in its high-affinity state (J. Li et al., 2017). Integrin binds a range of ligands via its extracellular domain, including the transmembrane protein Basigin (Bsg).

Bsg is an immunoglobulin superfamily glycoprotein, which serves as a transmembrane receptor in many different cell types. Bsg associates with many proteins serving diverse functions: inducing expression and activity of matrix metalloproteinases (Kaushik et al., 2015), binding and chaperoning monocarboxylate transporters to the membrane (Kirk et al., 2000; Wilson et al., 2002), and interacting with integrins in both vertebrates and invertebrates. In mice, Bsg and $\alpha$-integrin complex to induce differentiation in stem cells (Ozeki et al., 2015). Bsg and integrins also interact in human retinal pigmented epithelial cells (Priglinger et al., 2013). In hepatoma cells, the extracellular region of Bsg binds the extracellular head of the $\beta$-integrin subunit, facilitating signaling through focal adhesion kinase (Y. Li et al., 2012). In Drosophila, Bsg and integrin interact genetically in the embryo where they are involved in dorsal closure (Reed et al., 2004), and in the retina where they affect cellular structure (Curtin et al., 2005). Bsg serves additional functions in Drosophila, including regulating structure and function of synaptic boutons at the neuromuscular junction (NMJ) (Besse et al., 2007), promoting glial lipid droplet formation (Liu et al., 2017), mediating photoreceptor-glia interactions in the optic lamina (Curtin et al., 2007), and localizing monocarboxylate transporters to the salivary gland membrane (Velentzas et al., 2018). However, the role of Bsg in mediating peripheral glia development has not been determined.

Here, we investigate the role of Bsg in Drosophila glia during nervous system development, and in particular, its interaction with integrin. At the third instar larval stage, the nervous system is ensheathed by the perineurial glia, which interact with the overlying ECM. We found that Bsg is expressed in the perineurial glial in close proximity with integrin. Knockdown of Bsg in the perineurial glia results in reduced larval locomotion, significant shortening of the VNC, and compression of the glia and ECM in the peripheral nerves. Loss-of-function mutants of Integrin and Talin rescue the Bsg knockdown phenotype. Together, our results show that Bsg and Integrin interact in the glia to maintain the morphology of the nervous system.

\section{Materials and Methods}

Fly strains and genetics

The UAS-lifeact::GFP (Riedl et al., 2008), $m y s^{1}$ (Wright, 1960), $m y s^{11}$ (Wieschaus et al., 1984), and rhea ${ }^{1}$ (Brown et al., 2002) lines were gifts from Guy Tanentzapf. The GFP-tagged UAS-Bsg mutant constructs and $B s g^{1217}$ (Besse et al., 2007) were gifts from Anne Ephrussi, and $B s g^{265}$ (Curtin et al., 2005) was a gift from Bruce Reed. Bsg::cherry was generated using Recombinase-Mediated Cassette Exchange (Bateman et al., 2006) where a pseudo-exon carrying an in-frame mCherry coding sequence was spliced into the Bsg gene using the Minos-Mediated Integration Cassette system (Venken et al., 2011; Nagarkar-Jaiswal et al., 2015). DNA injection into Bsg ${ }^{M I 09180}$ flies and screening of mCherrypositive flies was done by Rainbow Transgenic Flies. The following fly strains were from the Bloomington Stock Center: repo-GAL4 (Sepp et al., 2001); UAS-mCD8::GFP (Lee and Luo, 1999); UAS-mCD8::RFP (E. Gavis, personal communication; http://flybase.org/reports/FBrf0207858); UAS-Dicer2 (Dietzl et al., 2007); Perlecan::GFP (Morin et al., 2001); Viking::GFP (Morin et al., 2001); and Bsg::GFP (Morin et al., 2001). 46F-GAL4 (Xie and Auld, 2011) was obtained from the Kyoto DGRC. Bsg-RNAi lines (GD989, GD15718) were from the VDRC. Mys-RNAi (1560R-1) was from the National Institute of Genetics in Japan. All RNAi experiments were conducted at $29^{\circ} \mathrm{C}$ with UAS-Dicer 2 in the background, unless otherwise specified. All larval tracking experiments were conducted at with larvae raised at $29^{\circ} \mathrm{C}$ with controls expressing UAS-Dicer2 and UAS- $m C D 8:: R F P$.

\section{Immunolabeling and image analysis}

Dissection and fixation of third instar larvae for immunofluorescence were performed as described previously (Sepp et al., 2000). For immunolabeling, larvae were fixed in 4\% PFA for 10-20 min. The following primary antibodies were used: mouse anti-Futsch (1:1000, Developmental Studies Hybridoma Bank), rat anti-tubulin YL1/2 (1:100, Cedarlane Laboratories), rabbit anti-HRP (1:500, Jackson ImmunoResearch Laboratories), anti-Bsg (1:100, gift from Anne Ephrussi), rabbit anti-zipper (1:2500, gift from Ken Prehoda), rabbit anti-GFP (1:500, Invitrogen), mouse anti- $\beta$ PS (1:10, CF.6G11) (Brower et al., 1984), rabbit antiNervana 2.1 (1:1000, Sigma Millipore), and mouse anti-acetylated tubulin (1:300, Sigma Millipore). Secondary antibodies were goat anti-mouse, goat anti-rat, or goat anti-rabbit conjugated with Alexa-488, Alexa-568, or Alexa-647 (1:300, Invitrogen). Fluorescent images were taken with a DeltaVision microscope (GE Healthcare) using a $20 \times$ (low magnification) or $60 \times$ (high magnification) oil-immersion objective (NA 1.4) at $0.2 \mu \mathrm{m}$ steps. Stacks were deconvolved with SoftWorx image processing software (SoftWorx) using a point spread function measured with a $0.2 \mu \mathrm{m}$ fluorescent bead (Invitrogen) in Vectashield (Vector Laboratories). Images were further processed and compiled using ImageJ (Schneider et al., 2012) or Fiji (Schindelin et al., 2012) and Adobe Illustrator (Adobe Systems). 3D reconstructions of nerves were generated using the Fiji ClearVolume plugin (Royer et al., 2015), and videos of the reconstructions were made using QuickTime screen-capture (Apple). For transmission electron microscopy (TEM) analysis, larvae were fixed in $4 \%$ formaldehyde and $3 \%$ glutaraldehyde, rinsed in $0.1 \mathrm{M}$ PIPES, postfixed in $1 \%$ osmium tetroxide, embedded in 1:1 acetonitrile:Spurr resin, and polymerized in Spurr resin. Thin sections $(50 \mathrm{~nm})$ were obtained with a Leica ultramicrotome and analyzed with a FEI Tecnai TEM operating at an accelerating voltage of $80 \mathrm{kV}$ (Bioimaging Facility, University of British Columbia).

Larval tracking. Larval tracking was performed using an adapted multiworm tracker and script (Swierczek et al., 2011; Nussbaum-Krammer et al., 2015; Petley-Ragan et al., 2016). Briefly, each tracking session involved placing 5-8 larvae on $90 \mathrm{~mm}$ high-contrast SYLGARD plates (Sigma Millipore) and tracking larval movement for $60 \mathrm{~s}$ using a Canon VIXIA HF R800 video camera (Canon). Videos were processed using the Fiji wrMTrck plug-in (Nussbaum-Krammer et al., 2015), which measured the average speeds of all larvae, and these values were used for analysis. Data from animals that could not be accurately tracked over time by the software were discounted.

Proximity ligation assay (PLA). The PLA assay was conducted using Duolink In Situ reagents as described previously (Wang et al., 2015; Samarasekera and Auld, 2018) with the adjustments that reaction volumes of PLA probe, ligation, and amplification mixes, and the incubation times were doubled and conducted in humid chambers placed on horizontal shaker at $37^{\circ} \mathrm{C}$. The antibody pair used was rabbit polyclonal anti-GFP and mouse monoclonal anti- $\beta$ PS. Negative controls were 
conducted with single primary antibodies alone. Larval fillets were mounted in Vectashield. The PLA images were captured using consistent exposure times and were analyzed in ImageJ. Mean fluorescence intensity of the PLA was calculated within an ROI with a fixed area using the Measure tool. All measurements were taken from the $z$ slice that fell in the middle of the $z$ stack.

Experimental design and statistical analysis For VNC length measurements, low-magnification images were stitched together using the Fiji Pairwise Stitching plug-in (Preibisch et al., 2009), and measurements were calculated using the linear measurement feature. All statistical tests were performed using GraphPad Prism 6.0 software. The difference in means between two groups was analyzed using a Student's $t$ test. Difference in means between multiple groups was analyzed using a one-way ANOVA and the Tukey post hoc test or the Dunnett's test for multiple comparisons. All graphs show the mean and SD. Values outside of $2 \mathrm{SD}$ from the mean were excluded from analyses. Detailed analysis of statistical tests is included in Results.

\section{Results}

Bsg is expressed in the CNS and PNS

To determine which cell populations within the CNS and PNS express Bsg, we examined the expression pattern of an endogenously GFP-tagged fusion protein, Bsg::GFP (Morin et al., 2001; Reed et al., 2004). In third instar larvae, Bsg::GFP is highly expressed at the NMJ, in the CNS neuropil, in axons exiting the neuropil, and in glial cells along the surface of both the CNS and PNS (Fig. 1A). Higher magnification of the peripheral nerves revealed that Bsg appeared to be expressed only in peripheral glia, not axons (Fig. 1C,D). Bsg::GFP fluorescence surrounding the CNS and along peripheral nerves was eliminated following expression of Bsg-RNAi using the pan-glial driver repo-GAL4 (Fig. $1 B$ ). However, Bsg::GFP fluorescence was retained at the NMJ, in the CNS neuropil, and in axons exiting the neuropil, consistent with previous studies showing that

Bsg is expressed in neurons (Besse et al., 2007). There was no Bsg:: GFP fluorescence seen along peripheral nerves after Bsg knockdown in glia, indicating that, within peripheral nerves, Bsg is primarily expressed by glia (Fig. $1 E, F$ ). We further confirmed these expression patterns using a second fusion protein, Bsg::cherry. Bsg::cherry was generated from a Bsg [MIMC] line using Recombinase-Mediated Cassette Exchange (Bateman et al., 2006) to generate an independent Bsg fusion protein. Both fusion proteins are homozygous viable. The expression patterns of the Bsg::GFP and Bsg::cherry fusion proteins matched in both the CNS and PNS. Both proteins were expressed in the neuropil, nerve roots exiting the neuropil, the glial cells ensheathing the entire nervous system, and at the NMJ (Fig. $1 G-K)$. We further verified the Bsgfusion proteins using an antibody against Bsg (data not shown).

\section{Glial knockdown of Bsg results in morphologic defects in the CNS} and PNS

To determine the role of Bsg in all Drosophila glia, we expressed Bsg-RNAi and a fluorescently tagged membrane marker (mCD8:: GFP) using the pan-glial driver repo-GAL4. The repo $>B s g-R N A i$ larvae survived to the third instar larval stage but died during pupal stages. Compared with control larvae, we found morphologic defects in both the CNS and PNS. The VNC was condensed, and there were sections of peripheral nerves with aberrant glial 


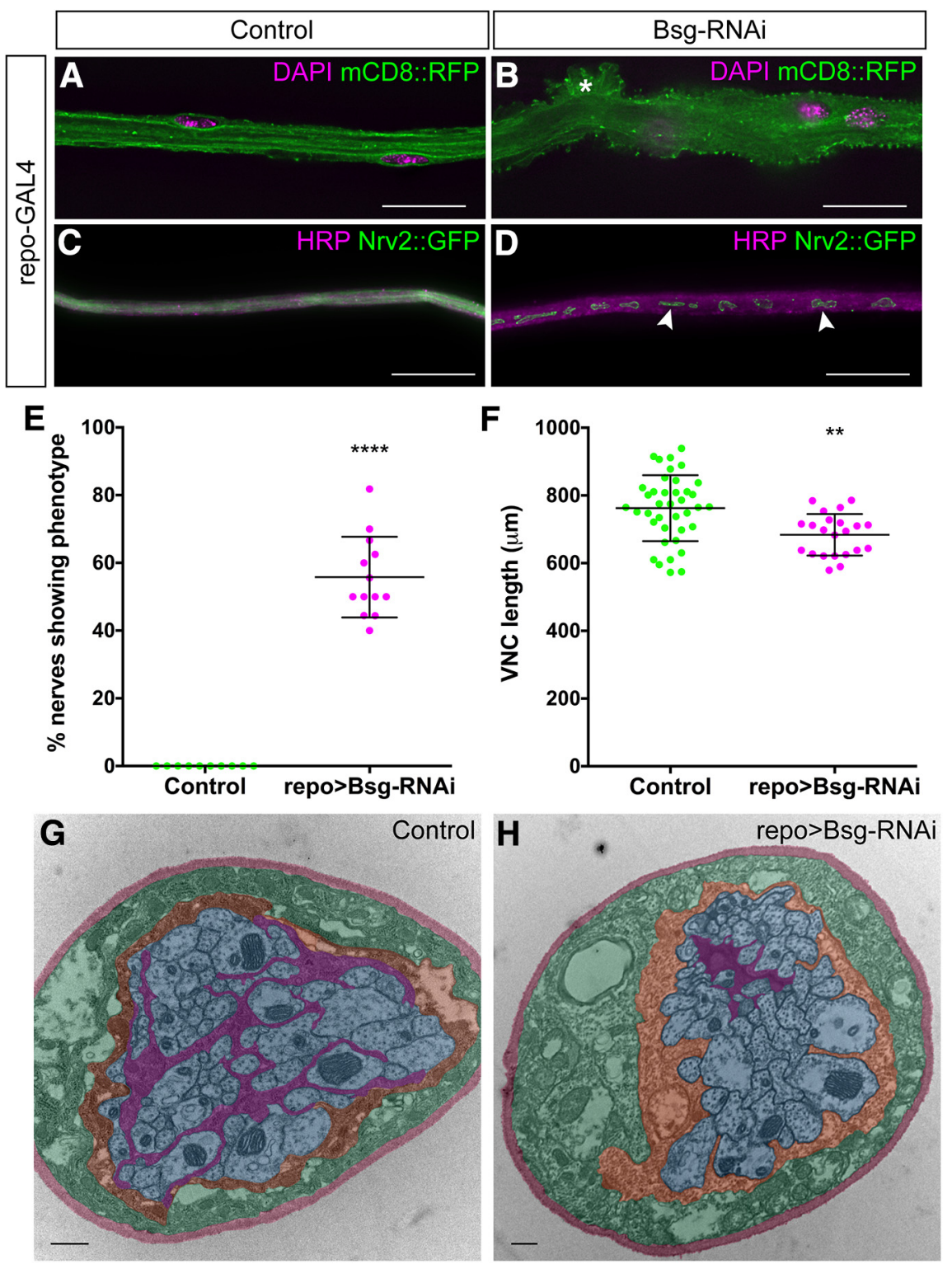

Figure 2. Pan-glial knockdown of Bsg causes morphologic defects and VNC shortening. $\boldsymbol{A}$, Peripheral nerve of third instar Drosophila larvae with all glial cells labeled using the pan-glial driver repo-GAL4 driving expression of membrane marker $\mathrm{mCD}$ ::RFP (green). $\boldsymbol{B}$, Knockdown of Bsg expression in all glial layers results in gross morphologic defects (asterisk) to the peripheral nerve. C, Peripheral nerve with wrapping glia labeled by Nrv2::GFP (green) and axons labeled using anti-HRP (magenta). D, Pan-glial Bsg knockdown results in fragmentation (arrowheads) of the wrapping glia (green). Scale bars, $15 \mu \mathrm{m}$. $\boldsymbol{E}$, Pan-glial Bsg knockdown using repo-GAL4 results in morphologic defects seen in $55.8 \%$ of nerves, which were never observed in control nerves (repo-GAL4) $\left({ }^{* * *} p<0.0001\right)$. F, Pan-glial Bsg knockdown results in a significant shortening of the VNC ( $\left.{ }^{* *} p=0.0022\right)$. G, TEM image showing cross-section through a WT peripheral nerve. Wrapping glia (false-colored magenta) fully ensheath and fasciculate the axons (blue). They are further ensheathed by the subperineurial glia (orange), perineurial glia (green), and the neural lamella (red). $\boldsymbol{H}$, Cross-section through repo $>$ Bsg-RNAi nerve shows that the axons (blue) are no longer ensheathed by the wrapping glia (magenta). Scale bars, $500 \mu \mathrm{m} .{ }^{* *} p<0.01,{ }^{* * *} p<0.0001$.

morphology and fragmented pieces of glial membrane within the interior of the nerve (Fig. $2 A-D$ ). The peripheral nerve in the third instar larva contains three glial layers: the innermost wrapping glia, the intermediate subperineurial glia, and the outermost perineurial glia, surrounded by a specialized ECM called the neural lamella. Peripheral nerves in control larvae are generally smooth in appearance with compact, flat glial layers (Fig. 2A). After Bsg knockdown in glia, $55.8 \%$ of peripheral nerves $(n=13$ larvae) demonstrated a ruffle-like phenotype, which was absent in control nerves $(n=10$ larvae) (repo $>m C D 8:: R F P$, Dicer2) (Fig. $2 A, B, E$ ). The location of the phenotype along the exterior of the nerve suggested that the perineurial glia were being affected. Perineurial glia also surround the larval CNS, which is comprised of the brain lobes and VNC. In control larvae, the length of the VNC was on average 762.2 $\pm 97.4 \mu \mathrm{m}$ $(n=39)$, whereas the VNCs of repo $>B s g$ $R N A i$ larvae were significantly shorter $\left(t_{(59)}\right.$ $=3.427, p=0.0020$, unpaired $t$ test) with an average length of $685.4 \pm 83.6 \mu \mathrm{m}(n=24)$ (Fig. $2 F$ ). The glial phenotype in the PNS may represent condensation in the peripheral nerve, similar to the condensation of the VNC in the CNS. The location of the fragmented glial membrane along the interior of the peripheral nerves suggested that the wrapping glia might also be affected. Therefore, we used Nrv2::GFP, a fusion protein endogenously expressed in wrapping glia, but not the subperineurial glia or perineurial glia, to assess the morphology of the PNS wrapping glia in controls and after Bsg knockdown (Fig. 2C,D). We confirmed that the wrapping glia were fragmented after Bsg knockdown. We further verified our findings using electron microscopy to assess the changes in the ultrastructure of the nerve. In controls (w1118), the wrapping glia surrounded the axons with extensive processes $(n=12)$ (Fig. 2G). In repo $>B s g-R N A i$ peripheral nerves, wrapping glia did not extend processes, whereas the subperineurial glia and axon structure was unaffected $(n=8)$ (Fig. $2 H)$. Our results suggest that Bsg plays a significant role in glia during the development and maintenance of the Drosophila nervous system, and is important for Drosophila survival during pupal stages.

\section{Bsgfunctions in the perineurial glia}

The expression of Bsg in the outer cell layers surrounding the CNS and PNS suggested that it is present in the perineurial glia. To test the localization and function of Bsg in the glia, we knocked down Bsg expression specifically in each glial layer using RNAi and the driver lines nrv2GAL4 (wrapping glia) (Sun et al., 1999), Gli-GAL4 (subperineurial glia) (Sepp and Auld, 1999), and 46F-GAL4 (perineurial glia) (Xie and Auld, 2011) in the background of Bsg::GFP. Knockdown in the wrapping glia $(n r v 2>B s g-R N A i)$ or the subperineurial glial ( $G l i>B s g-R N A i$ ) did not generate any phenotypes (data not shown). However, we found that, similar to panglial knockdown, Bsg::GFP expression surrounding the CNS and PNS was eliminated using 46F-GAL4 (Fig. 3A-D). Bsg::GFP fluorescence remained in the neuropil, in axons exiting the neuropil, and at the NMJ (Fig. 3A,B). We found that Bsg knockdown using $46 F-G A L 4$ was sufficient to cause significant shortening of the $\operatorname{VNC}\left(t_{(56)}=5.962, p<0.0001\right.$, unpaired $t$ test) (Fig. $\left.3 G\right)$. Control larvae had an average VNC length of $763.7 \pm 100.2 \mu \mathrm{m}(n=23)$, whereas $46 F>B s g-R N A i$ larvae had an average VNC length of 

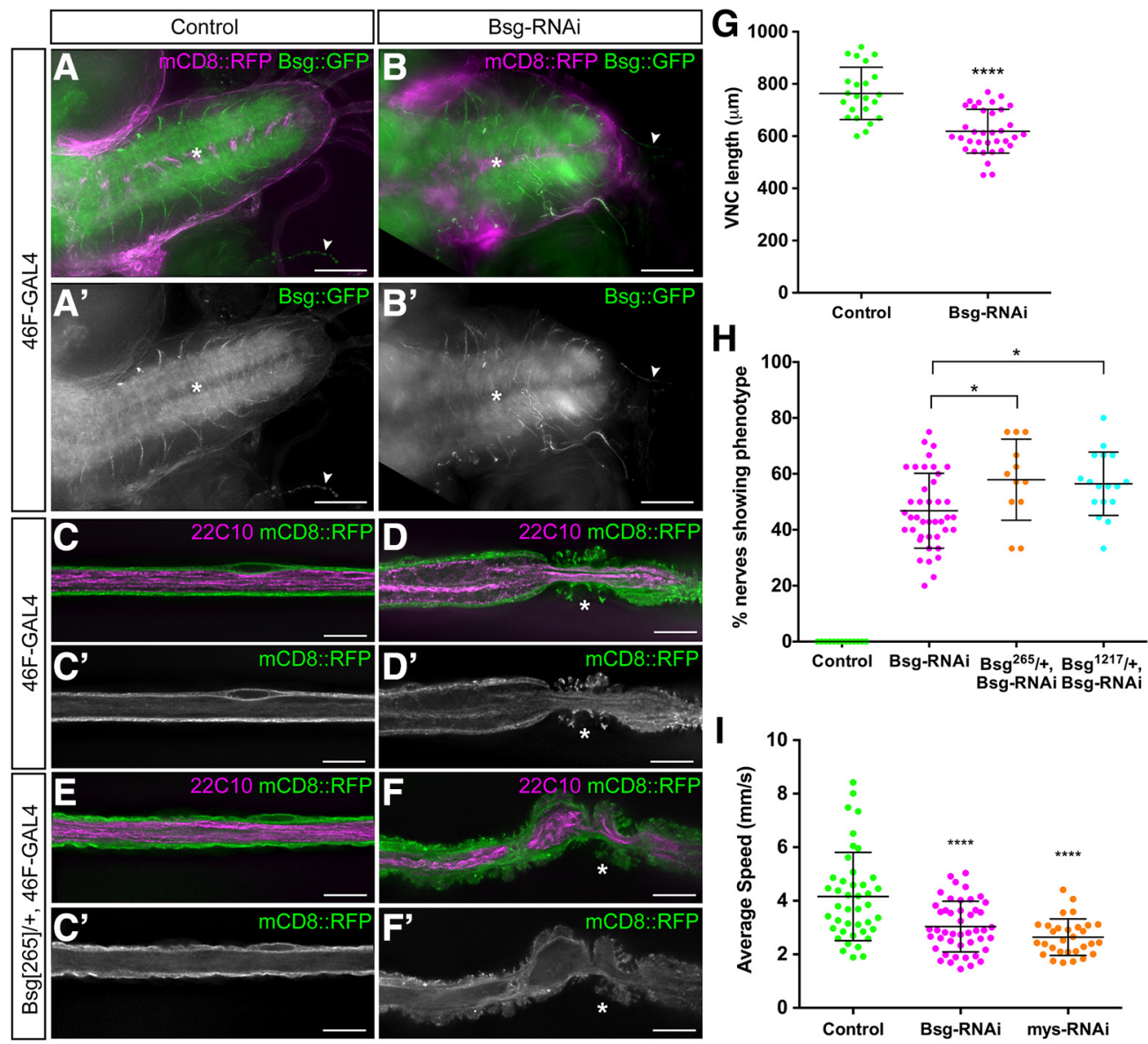

Figure 3. Perineurial glia-specific knockdown of Bsg causes VNC shortening, peripheral glial abnormalities, and reduced larval locomotion. $A, B$, The VNC of third instar Drosophila larvae expressing endogenously tagged Bsg::GFP (green) and 46F-GAL4 driving expression of mCD8::RFP (magenta). Knockdown of Bsg in the perineurial glia using Bsg-RNAi results in shortening of the VNC (B) compared with controls $(\boldsymbol{A})$ and a loss of Bsg expression in the glia ensheathing the CNS and PNS while expression is retained in the neuropil (asterisks) and the NMJs (arrowheads). Scale bar, $50 \mu \mathrm{m}$. $\boldsymbol{C}-\boldsymbol{F}$, Peripheral nerve of third instar 46F $>m C D 8:: R F P$ (green) larvae with axons labeled using anti-Futsch/22C10 (magenta). Bsg knockdown results in compression of the glia (D, asterisk), and knockdown in a heterozygous Bsg mutant (Bsg[265]) exacerbates the phenotype ( $\boldsymbol{F}$, asterisk). Scale bars, $15 \mu \mathrm{m}$. $\boldsymbol{G}$, Bsg knockdown results in significant shortening of the VNC $\left({ }^{* * * *} p<0.0001\right)$ compared with control (46F-GAL4). H, Penetrance of glial compression was significantly increased in the background heterozygous for Bsg[265] ( $\left.{ }^{*} p=0.0088\right)$ compared with control (46F-GAL4) and Bsg-RNAi alone. I, Knockdown of Bsg (magenta) and the integrin $\beta$-subunit myospheroid (mys) (orange) significantly reduced larval locomotion ( $\left.{ }^{* * * *} p<0.001\right)$ compared with control $46 \mathrm{~F}>$ Dicer2 (green). ${ }^{*} p<0.05,{ }^{* * *} p<0.0001$

$618.6 \pm 83.9 \mu \mathrm{m}(n=35)$. Additionally, we found that knocking down Bsg in the perineurial glia resulted in the same aberrant morphologic defects along the peripheral nerves (Fig. 3D). Thus, much of the phenotype associated with Bsg knockdown in all glia can be attributed to the lack of Bsg specifically in the perineurial glia. When we repeated the knockdown in the background of a heterozygous loss-of-function mutation $B s g$ [265], the penetrance of the nerve phenotype was significantly enhanced $\left(F_{(3,79)}=69.23\right.$, $p=0.0318$, ANOVA, Tukey post hoc test) (Fig. $3 H$ ) from appearing in $46.8 \%$ of nerves ( $n=41$ larvae) in the $46 F>B s g-R N A i /+$ larvae to $57.9 \%$ of nerves ( $n=12$ larvae) in the $46 F>B s g-R N A i / B s g$ [265]/ + larvae. We confirmed this result using a second loss-of-function mutant Bsg[1217], which also significantly enhanced the phenotype to appearing in $56.5 \%$ of nerves $(n=17$ larvae $)\left(F_{(3,79)}=69.23\right.$, $p=0.0330$, ANOVA, Tukey post hoc test). We used the Bsg-RNAi line GD989 for all subsequent knockdown experiments; but to control for off-target effects, we tested a second Bsg-RNAi line, GD15718. This line, when driven by $46 F-G A L 4$ with UAS-Dicer2, resulted in the phenotype appearing in $44.1 \%$ of peripheral nerves ( $n=12$ larvae), confirming that our results were indeed a result of loss of Bsg expression. Finally, we wanted to determine whether the morphologic defects of Bsg knockdown in glia affected the function of the nervous system. To test this, we examined the mobility of third instar larvae using an adapted multiworm tracker. We found that the average speed of larvae was significantly reduced from $4.3 \pm 1.8 \mathrm{~mm} / \mathrm{s}(n=43)$ in control larvae $(46 F>$ Dicer2) to $3.1 \pm 1.1 \mathrm{~mm} / \mathrm{s}(n=47)$ after Bsg knockdown $\left(46 F>\right.$ Dicer2, Bsg-RNAi) $\left(F_{(2,218)}=7.284, p<0.001\right.$, ANOVA, Tukey post hoc test) (Fig. 3I). As a control, we knocked down the integrin $\beta$-subunit myospheroid (46F>mys$R N A i$ ), which we had previously shown disrupted the ability of perineurial glia to wrap the peripheral nerve (Xie and Auld, 2011). Loss of integrin also disrupted larval locomotion, causing a significant reduction in speed to $2.6 \pm 0.6 \mathrm{~mm} / \mathrm{s} \quad(n=38)$ $\left(F_{(2,218)}=7.284, p<0.001\right.$, ANOVA, Tukey post hoc test) (Fig. $3 I$ ). Our results demonstrate that Bsg is a critical regulator of perineurial glial morphology.

Bsg knockdown in the perineurial glia affects the overlying ECM and underlying septate junctions

In order to further characterize the ruffle-like glial phenotype, we investigated the morphology of the overlying ECM in the Bsg knockdown background using endogenously tagged ECM component Perlecan::GFP. Normally, the ECM appears as a 


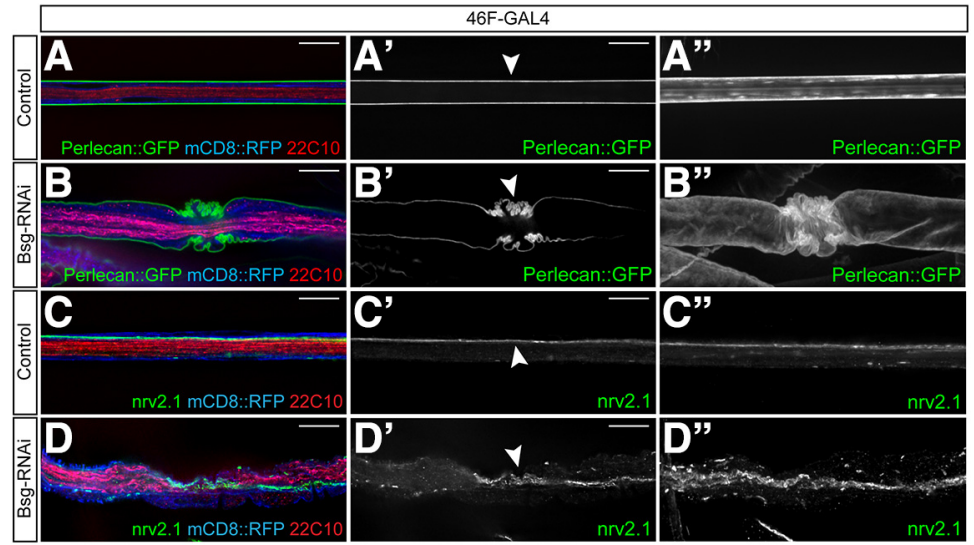

Figure 4. Perineurial glial constriction causes constriction of both the overlying ECM and underlying septate junctions. $A, B$, Peripheral nerves of third instar larvae expressing endogenously tagged ECM component Perlecan::GFP (green) and 46F-GAL4 driving expression of mCD8::RFP (blue) with axons labeled using anti-Futsch/22C10 (red). Bsg knockdown in the perineurial glia results in compression of the ECM in regions of glial constriction (arrowhead). Projections of 3D-reconstructed nerves $\left(\boldsymbol{B}^{\prime \prime}\right)$ show convoluted infolding of the ECM in $46 \mathrm{~F}>B s g-R N A i$ animals compared with the smooth sheath seen in control nerves $\left(\boldsymbol{A}^{\prime \prime}\right)$. C, D, Peripheral nerves from 46F>mCD8::RFP larvae labeled using an antibody against core septate junction component Nervana 2.1 (nrv2.1, green). Septate junctions run laterally along the length of the nerve between the opposing membrane of the subperineurial glia in control animals $\left(\boldsymbol{C}^{\prime}\right.$, arrowhead). In regions of glial compression, the septate junction strands appear convoluted ( $\boldsymbol{D}^{\prime}$, arrowhead), but $3 \mathrm{D}$ reconstruction shows that the strands remain continuous $\left(\boldsymbol{D}^{\prime \prime}\right)$. Scale bars, $15 \mu \mathrm{m}$.

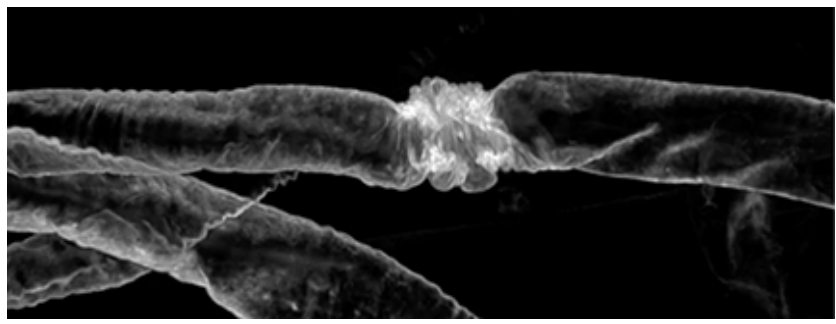

Movie 1. 3D reconstruction of ECM sheath shows accordion-like constriction. A peripheral nerve from a third instar larvae expressing endogenously tagged ECM component Perlecan:: GFP and 46F-GAL4 driving expression of Bsg-RNAi in the perineurial glia was reconstructed in 3D from a $z$ stack. In the region of glial compression, the ECM sheath appears highly convoluted and shows deep infolding compared with adjacent regions of the nerve where the sheath is smooth. [View online]

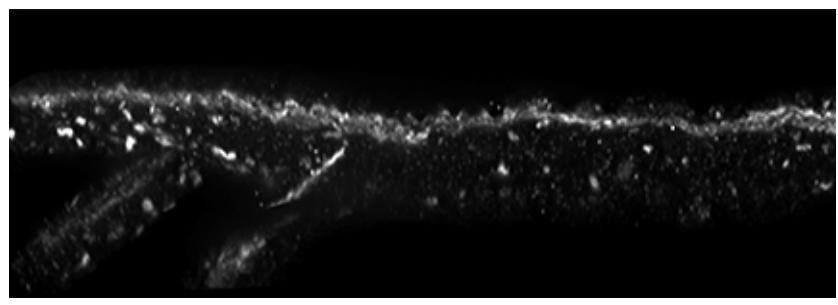

Movie 2. 3D reconstruction of the septate junction strands shows convolution but no discontinuities in regions of glial compression. A peripheral nerve from a third instar larvae expressing 46F-GAL4 driving Bsg-RNAi in the perineurial glia with the septate junctions (SJs) labeled using an antibody against core SJ component Nervana 2.1 was reconstructed in 3D from a $z$ stack. The SJs between opposing membranes of the subperineurial glia appear convoluted in regions of glial compression, but the strands remain continuous. [View online]

smooth, continuous sheath around the exterior of the nerve (Fig. 4A). We discovered that the ECM was highly convoluted in regions where the perineurial glial phenotype was seen (Fig. 4B), indicating that the glial membrane and overlying ECM are undergoing an inward constriction rather than membrane ruffling. We created $3 \mathrm{D}$ reconstructions of the nerves from the image stacks to better visualize the phenotype (Movie 1). The constriction phenotype became clear in the 3D-reconstructed nerves, where the ECM showed infolding in the areas where the glial phenotype was seen (Fig. $4 B^{\prime \prime}$ ), in contrast to the control nerves where the ECM forms a smooth cylinder around the nerve (Fig. $\left.4 A^{\prime \prime}\right)$. Similar results were seen when visualizing another ECM component, collagen IV, using Viking::GFP (data not shown). Having determined that Bsg knockdown caused constriction of the perineurial glial membrane and overlying ECM, we investigated whether the morphology of the underlying subperineurial glia was also affected. The subperineurial glia form septate junctions between their opposing membranes, so that the junction appears as a single line running laterally along the length of the nerve (Fig. 4C). We labeled the junctions using an antibody against the core septate junction component Nervana 2.1 to determine whether the morphology of the junction, and therefore the subperineurial glia, was affected by the perineurial glial constriction. In the regions of glial constriction, the septate junction strands have a sinuous appearance following the overlying perineurial constriction (Fig. 4D). The nerves were reconstructed in $3 \mathrm{D}$, and the images indicate that the septate junction strands do not become discontinuous ( $n=10$ larvae) (Fig. 4D'"; Movie 2), suggesting that constriction of the overlying perineurial glia disrupts the morphology but not the integrity of the junctions. These data indicate that the morphologic deformation of the perineurial glia is relayed both to the overlying ECM and to the underlying subperineurial glia.

Glial constriction is limited to the nerve extension region (NER) The glial constriction phenotype we identified in the Bsg knockdown animals had not been identified previously in other genetic backgrounds, so we sought to define further where the phenotype appeared within the peripheral nerve. We scored the location of the areas of constriction as being either directly proximal to the VNC, within the NER, or within the muscle field area (MFA) (Fig. 5). We discounted any regions of constriction seen directly proximal to the VNC because we occasionally observed aberrant morphology of nerves in this region of control animals $(1.4 \%, n=12$ larvae), but constriction was never observed in the NER in controls. For the constrictions generated by Bsg-RNAi, we analyzed whether the phenotype occurred within a specific region of the NER. Mapping of the phenotype location within the peripheral nerves revealed that the regions of glial constriction were found along the NER of the abdominal nerves A3-A8, but not A1-A2, without any clear pattern of distribution (Fig. 5). Therefore, while A1-A2 appear to be resistant, there does not seem to be any specific area of the other abdominal nerves that is more susceptible to morphologic aberration.

\section{Glial constriction is associated with cytoskeleton disruption}

Bsg knockdown in the glia causes very apparent morphologic changes to the glia and overlying ECM. We sought to determine 


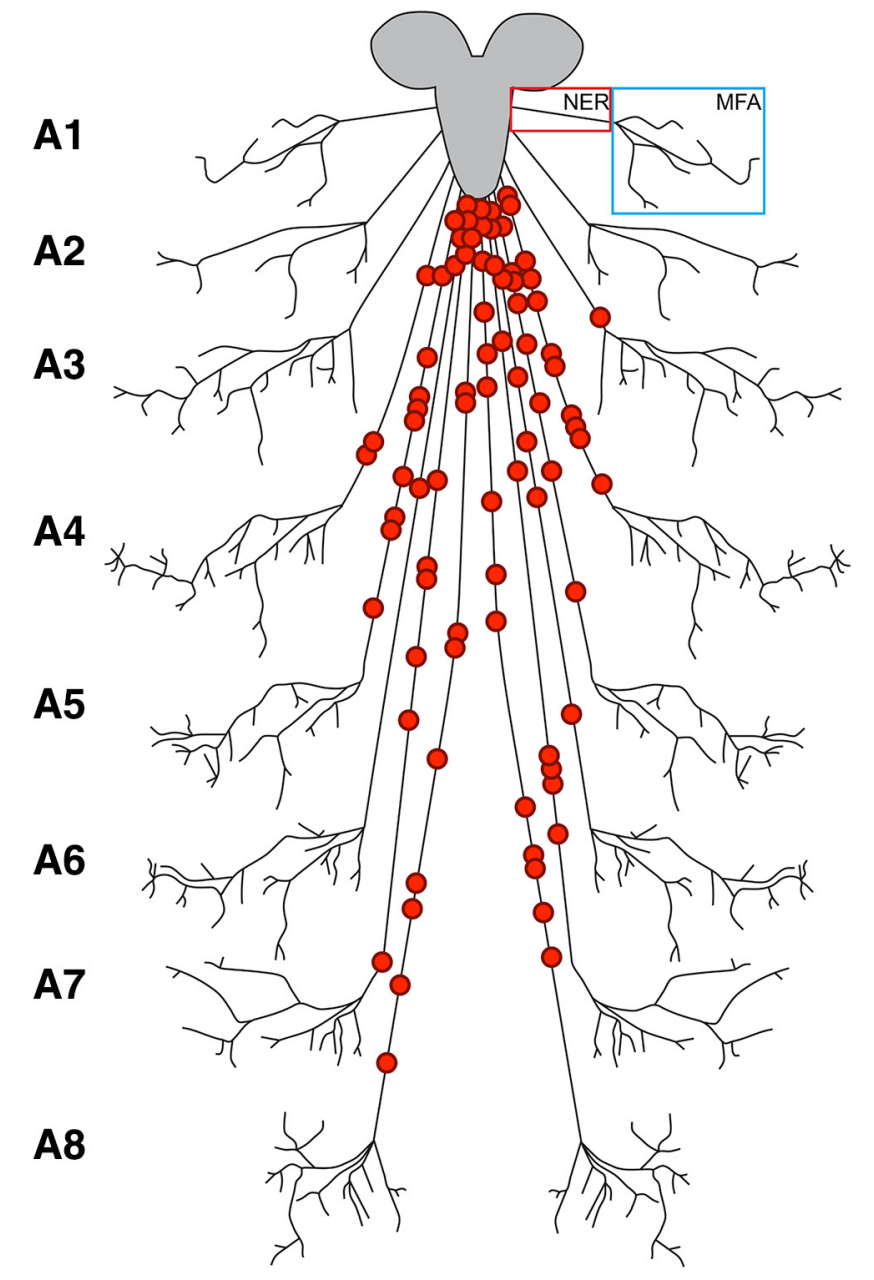

Figure 5. Schematic showing random distribution of glial constrictions throughout the NER. Regions of glial constriction were scored for their approximate location on the abdominal nerves A1-A8. All glial constrictions were found within the NER, and none was found in the MFA of any nerve. Constrictions were found mainly on the longer nerves (A4-A8) without any clear pattern of distribution.

whether these aberrations in cell morphology had an effect on the underlying cellular architecture. The ECM links to the cellular cytoskeleton via the integrin-based focal adhesion complexes transmitting mechanical signals between the intracellular compartment and the overlying ECM (Sun et al., 2016). We hypothesized that, due to the changes in glial and ECM morphology, there may also be changes to cytoskeletal architecture. In order to assess whether the glial actin cytoskeleton was affected by the Bsg knockdown phenotype, we drove expression of filamentous actin marker lifeact::GFP with 46F-GAL4, specifically labeling filamentous actin in the perineurial glia. In these cells, the actin filaments are usually seen as continuous strands running laterally along the length of the nerve (Fig. $6 A$ ). We found that, in areas of glial constriction, there were clear discontinuities in the actin cytoskeleton, indicative of filament breakage (Fig. 6B; Movie 3). These actin breakages were seen in $95 \%$ of the glial compression areas ( $n=18$ larvae). We also observed accumulation of GFP-positive puncta at the tips of the constricted ruffles, suggestive of broken actin filaments. To further investigate the effects of glial constriction on the cytoskeleton, we analyzed the appearance of myosin in the glia when Bsg was knocked down. Nonmuscle myosin II is expressed in the cells of the nervous system in both vertebrates and invertebrates, where it associates with actin (Hatan et al., 2011). We immunolabeled for Zipper, the heavy chain of Drosophila nonmuscle myosin II, in a $46 F>$ lifeact::GFP background to see how myosin and actin interact in the glial constriction region. We found gaps in Zipper labeling coincident with regions of glial constriction (Fig. $6 D$ ) (84\% compressions, $n=8$ larvae). Microtubules are another important cytoskeletal component; and although their function has not been characterized in the Drosophila glial cells, in vertebrates they play essential roles in morphology and trafficking in radial glia and astrocytes $(\mathrm{H}$. Li et al., 2003; Potokar et al., 2007). We immunolabeled for tubulin in a $46 F>$ lifeact::GFP background and found that there were large gaps in tubulin labeling in the regions of glial constriction (78\% of glial compressions, $n=6$ larvae), in contrast to the continuous microtubules seen along the length of the control nerve (Fig. 6E,F). The gaps in the microtubules always matched the discontinuities in actin filaments (data not shown). We also labeled for acetylated tubulin in this background, as acetylated microtubules are generally more stable and resistant to stressors (Janke and Montagnac, 2017). The acetylated microtubules also showed large gaps in glial compression regions (73\% of glial compressions, $n=6$ larvae). Finally, we labeled for the core focal adhesion component $\beta$-integrin (myospheroid) in the $46 \mathrm{~F}>$ lifeact::GFP background to determine whether integrin colocalized with the actin::GFP-positive puncta located in the tips of the glial constrictions. In control nerves, $\beta$-integrin is distributed uniformly along the glial sheath (Fig. $6 G$ ). In regions of glial compression, we found that $\beta$-integrin localized to some, but not all, of the constriction tips similarly to actin (Fig. $6 H$ ). Collectively, these data suggest that Bsgknockdown in the perineurial glia causes disruption to the glial cytoskeleton and that this maybeassociated with the integrin-based focal adhesions.

Bsg and integrin bind at the surface of perineurial glia Bsg has been previously demonstrated to interact with integrins in Drosophila (Reed et al., 2004; Curtin et al., 2005, 2007) and vertebrate models, including mouse (Berditchevski et al., 1997; Ozeki et al., 2015) and human cells (Y. Li et al., 2012; Priglinger et al., 2013). Integrin knockdown in the perineurial glia results in severe morphologic defects, including the absence of glial process extension around peripheral nerves (Xie and Auld, 2011). We observed that integrin knockdown in perineurial glia also resulted in decreased mobility (Fig. $3 H$ ). We also found that Bsg knockdown caused defects to both the ECM and the underlying glial cytoskeleton, components that are linked by the integrinbased focal adhesion complexes. We therefore hypothesized that Bsg is interacting with integrin in the perineurial glia. Using immunolabeling of the common integrin $\beta$-subunit, $\beta \mathrm{PS} / \mathrm{myo}$ spheroid, we found that Bsg::GFP and integrin colocalize in the perineurial glia (Fig. 7A). To confirm this colocalization, we performed a PLA that detects protein interactions within $40 \mathrm{~nm}$ in fixed tissues (Söderberg et al., 2006). We observed a positive PLA reaction between Bsg::GFP and integrin using anti-GFP and anti- $\beta$ PS antibodies that recognized the respective epitopes on the extracellular side of the glial membrane (Fig. $7 B$ ). Negative controls where one antibody was absent failed to show positive PLA reactions (Fig. 7C). Positive controls using Viking::GFP with anti-GFP and anti- $\beta$ PS antibodies confirmed that ECM components had positive PLA reactions with integrin as expected (Fig. 7D), and this reaction was abolished in the absence of the anti-GFP antibody (Fig. 7E). These results were further confirmed using Perlecan::GFP (Fig. 7H). Positive PLA reactions between the ECM and integrin were retained when Bsg was knocked down $(46 F>B s g-R N A i)$ (Fig. $7 F)$, indicating that 


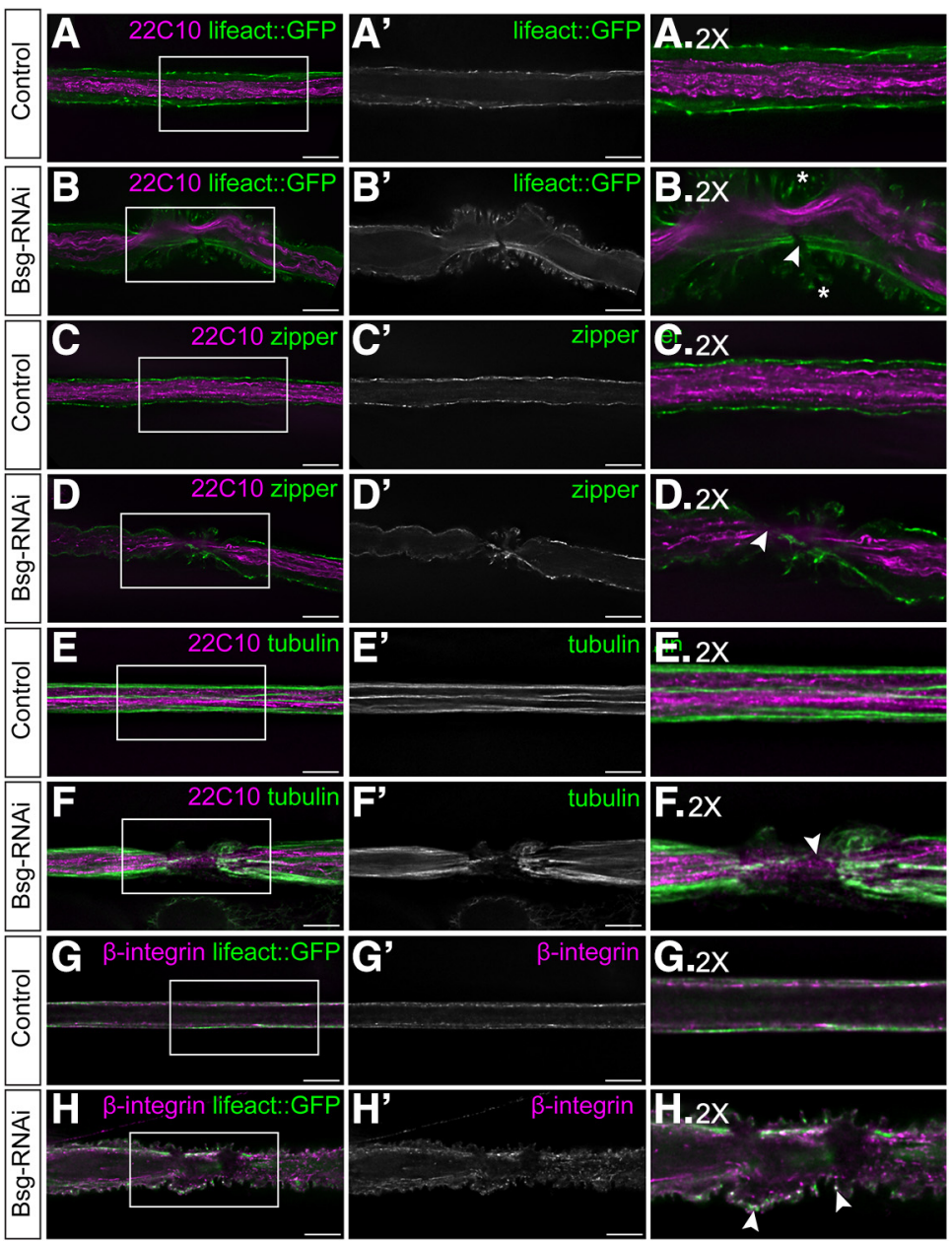

Figure 6. Glial compression is associated with discontinuities in cytoskeleton components. $\boldsymbol{A}, \boldsymbol{B}$, Peripheral nerves of third instar larvae expressing filamentous actin marker lifeact::GFP (green) driven by 46F-GAL4, with axons labeled using anti-Futsch/22C10 (magenta). Actin filaments show discontinuities in the regions of glial compression ( $\boldsymbol{B}$, arrowhead), and lifeact::GFP-positive puncta are accumulated at the tips of the compressions (asterisks). C, D, Nerves labeled with antiFutsch (magenta) and an antibody against nonmuscle myosin II (zipper, green) to show distribution of myosin motors. Regions of glial compression were associated with gaps in labeling of myosin ( $\boldsymbol{D}$, arrowhead). $\boldsymbol{E}, \boldsymbol{F}$, Nerves labeled with anti-Futsch (magenta) and anti-tubulin to show morphology of microtubules (green). In regions of glial compression, microtubules appear fragmented $(\boldsymbol{F}$, arrowhead). $\mathbf{G}, \boldsymbol{H}$, Nerves expressing lifeact::GFP (green) and labeled with an antibody against the $\beta$-subunit of the integrin heterodimer (magenta). Integrin is localized to the focal adhesion complexes, which are distributed uniformly along the length of the glial sheath. In regions of glial compression, $\beta$-integrin is found within the compressed glial cells and at the tips of some the compressions alongside broken actin filaments ( $\boldsymbol{H}$, arrowheads). Side panels represent $2 \times$ digital magnifications of the indicated regions in the corresponding panels. Scale bars, $15 \mu \mathrm{m}$. loss of Bsg does not reduce the presence of integrin at the membrane and the proximity to the ECM. The positive PLA reaction between the ECM and integrin was reduced when $\beta$-integrin expression was knocked down in the perineurial glia $(46 F>m y s-R N A i)$ (Fig. $7 G, H$ ). When the PLA fluorescence was quantified (Fig. $7 \mathrm{H}$ ), all integrin-ECM interactions were significantly greater than controls lacking the anti- $\beta$ PS antibody, except when $\beta$ PS was reduced using $\operatorname{RNAi}\left(F_{(5,143)}=\right.$ 4.337, $p=0.0011$, ANOVA, Dunnett post hoc test).

The positive PLA reactions indicated that Bsg and integrin are located within close proximity and may associate on the surface of perineurial glia. Crystal structure analysis of vertebrate Bsg indicated that the extracellular membrane-proximal domain of Bsg binds integrin in trans conformation (Y. Li et al., 2012). However, as Bsg serves a wide range of functions in multiple different tissues, we sought to determine which domains within the Bsg structure are responsible for interacting with integrin in the Drosophila glial cells. To answer this, we expressed a range of mutant Bsg constructs tagged with GFP (Besse et al., 2007) (Fig. 8A) using 46F-GAL4 and performed PLA using anti-GFP and anti- $\beta$ PS antibodies. The control construct UAS-Bsg:: GFP, when expressed in the perineurial glia, had a positive PLA reaction with $\beta$-integrin (Fig. $8 B$ ). Specific residues (KRR) in the intracellular membrane-proximal domain of Bsg have been demonstrated to be important for NMJ structure in Drosophila (Besse et al., 2007); however, we found that, when these residues were mutated to NGG (Bsg::GFPNGG), there was still a positive PLA reaction between integrin and Bsg (Fig. 8C). The transmembrane domain of Bsg contains a highly conserved charged glutamate residue, which has been shown to be critical for its binding to some transmembrane proteins, such as monocarboxylate transporters in COS cells and Xenopus ooctyes (Manoharan et al.,

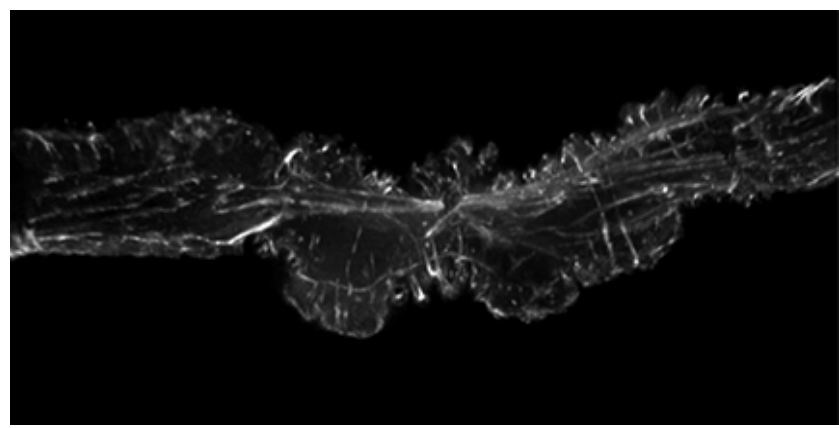

Movie 3. 3D reconstruction of the perineurial glial actin cytoskeleton shows breakage of actin filaments in regions of glial compression. A peripheral nerve from a third instar larvae expressing 46FGAL4 driving expression of fluorescent actin marker lifeact::GFP and Bsg-RNAi in the perineurial glia was reconstructed in $3 \mathrm{D}$ from a z stack. In regions of glial compression, the actin filaments appear discontinuous, and GFP-positive puncta accumulate in the tips of the compressions. [View online]
2006). The Bsg::GFP-CD2 mutant, a chimeric Bsg construct composed of two Ig domains of Bsg, fused to the transmembrane and cytoplasmic domains of rat CD2, still had a positive PLA with integrin (Fig. 8D). Finally, we determined that removing the short $\mathrm{C}$-terminal intracellular domain of Bsg (Bsg::GFP- $\Delta$ intra) did not affect the PLA interaction with integrin (Fig. 8E). When the fluorescence intensity of the PLA reactions was quantified, there was a significant PLA reaction with each of the Bsg::GFP constructs compared with the antibody-absent control $\left(F_{(4,109)}=12.19, p<0.0001\right.$, ANOVA, Dunnett post hoc test) (Fig. $8 F$ ). Together, these findings demonstrate that the extracellular portion of Bsg contains the domains necessary for interactions with integrin.

To further test whether the interaction between Bsg and integrin at the membrane may underlie the glial constriction phenotype, we used the mutant Bsg constructs to attempt to 

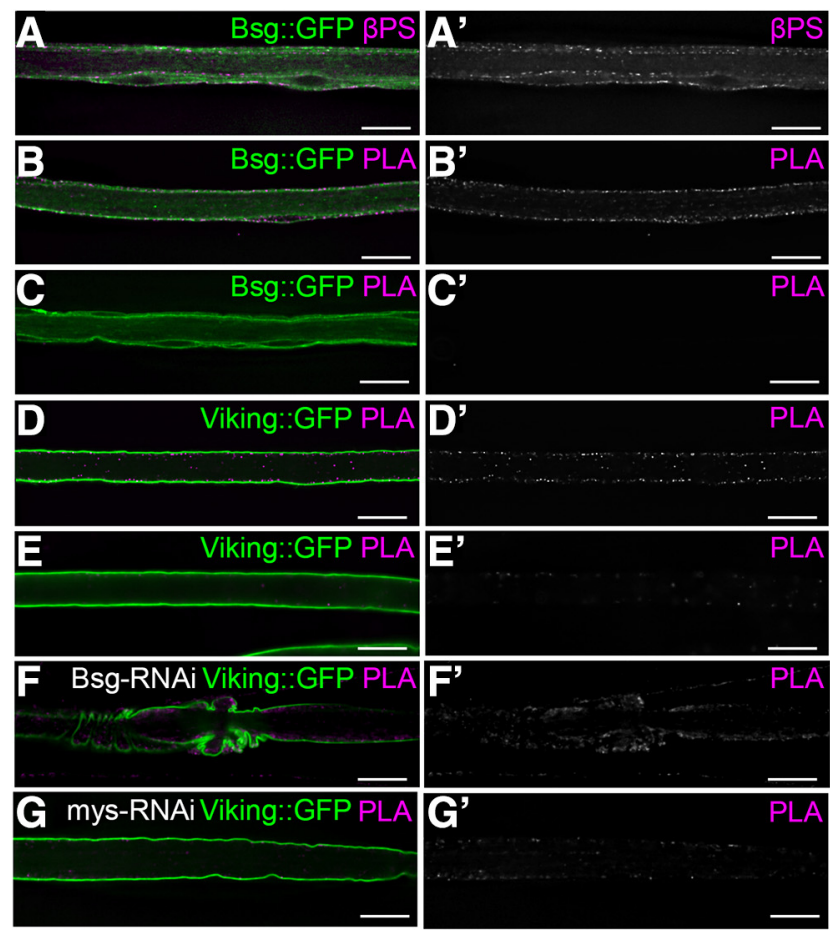

\section{H}

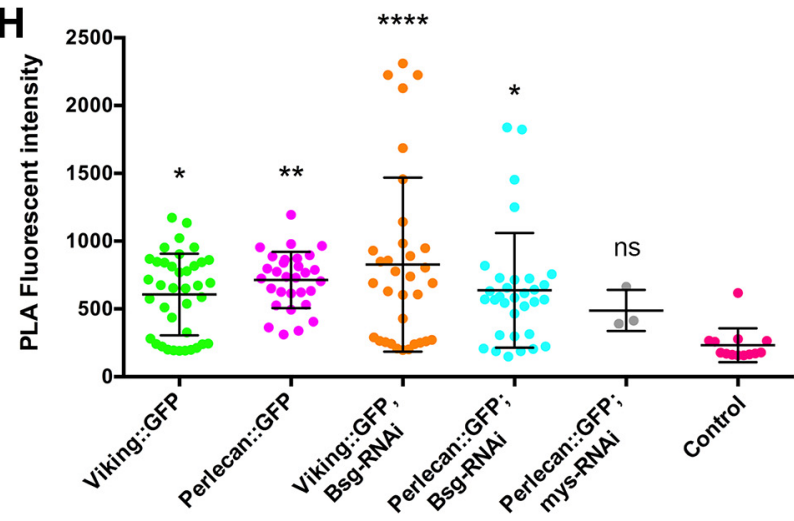

Figure 7. Integrin is in close proximity to the extracellular domain of Bsg and the ECM in the perineurial glia. $A$, Peripheral nerve of Bsg::GFP larvae with integrin $\beta$-subunit labeled using anti- $\beta$ PS (magenta). Bsg and integrin are coexpressed in the perineurial glia (arrowheads). $\boldsymbol{B}$-J, PLA (magenta) with GFP tagged proteins (green) and the $\beta$ PS integrin subunit. $B$, PLA using anti-GFP and anti- $\beta$ PS antibodies shows that Bsg::GFP and integrin are found in close proximity $(<40 \mathrm{~nm})$ in the perineurial glia. $C$, Negative control of PLA experiment where anti-GFP antibody was absent shows no PLA. $\boldsymbol{D}$, Positive control of PLA experiments showing ECM component collagen-IV (Viking::GFP) is found in close proximity to integrin. $\boldsymbol{E}$, Negative control where the anti-GFP antibody was absent shows no PLA. F, Positive PLA between collagen-IV (Viking::GFP) and integrin occurs in knockdown of Bsg in the perineurial glia (46F>Bsg-RNAi). G, Control where $\beta$-integrin was knocked down in the perineurial glia (46F>mys-RNAi) shows reduced PLA with Viking::GFP. Scale bars, $15 \mu \mathrm{m}$. $\boldsymbol{H}$, Quantification of the PLA reaction fluorescence intensity between the $\beta$ PS integrin subunit and ECM components Viking and Perlecan tagged with GFP. Fluorescence intensity was significantly higher for all reactions compared with control (Viking::GFP minus the anti- $\beta$ PS antibody) with the exception of when $\beta$-integrin (mys-RNAi) was knocked down. From left to right: Viking:: GFP $+\beta$ PS, ${ }^{*} p=0.0208$; Perlecan::GFP $+\beta$ PS, ${ }^{* *} p=0.0023$; Viking::GFP $+\beta$ PS, BsgRNAi, ${ }^{* * * *} p<0.0001$; Perlecan::GFP $+\beta$ PS, Bsg-RNAi, ${ }^{*} p=0.0130$; Perlecan:: $\mathrm{GFP}+\beta \mathrm{PS}$, mys-RNAi, ns $p=0.7835 .{ }^{*} p<0.05,{ }^{* *} p<0.01$.

rescue the compression phenotype. We knocked down Bsg:: cherry in the perineurial glial using cherry-RNAi in a Bsg[265]/ + background, to increase the effectiveness of the Bsg knockdown in the absence of UAS-Dicer2. To test the ability of the
Bsg transgenes to rescue, we coexpressed the GFP-tagged mutant Bsg constructs UAS-Bsg::GFP-NGG and UAS-Bsg:: GFP-CD2, with the full-length UAS-Bsg::GFP as a control (Fig. $8 G$ ). We also expressed UAS-mCD8::GFP to control for the number of UAS transgenes. Knockdown of Bsg::cherry using cherry-RNAi resulted in glial compression in $40.2 \%$ of nerves (Bsg[265],Bsg::cherry,46F>cherry-RNAi) ( $n=4$ larvae). However, expression of the control (Bsg::GFP) and mutant (Bsg::GFP-NGG and Bsg::GFP-CD2) constructs in this background completely rescued the phenotype $\left(F_{(4,27)}=\right.$ 49.25, $p<0.0001$, ANOVA, Dunnett post hoc test) (Fig. 8G). These results indicate that the extracellular domain, but not the transmembrane or intracellular domains, is necessary for maintenance of glia-ECM integrity.

Bsg knockdown in an integrin mutant background rescues constriction phenotype

The ECM and glial cytoskeletal phenotypes seen in the Bsg knockdown led us to hypothesize that the glial constriction phenotype may be mediated by the integrin-based focal adhesions. The focal adhesions link the ECM to the underlying cytoskeleton via the transmembrane integrin dimer and associated proteins, including Talin (Sun et al., 2016). We hypothesized that the glial constriction phenotype is a result of an increase in integrin-based adhesion between the glial membrane and the overlying ECM, causing both the glia and ECM to constrict inwards. To test this hypothesis, we knocked down Bsg in a heterozygous integrin mutant background using the loss-of-function $\beta$-integrin mutant $m y s^{11}$. We did not see any glial compression when Bsg was knocked down in the $m y s^{11} /+$ heterozygous background $\left(m^{11} s^{11} /\right.$ $+; 46 \mathrm{~F}>B s g-R N A i, n=11$ larvae), in contrast to the $46 \mathrm{~F}>B s g$ RNAi animals ( $n=20$ larvae) where compression was seen throughout the nerves (Fig. 9D,E,H). The VNC compression phenotype was also rescued in the $m y s^{11} /+; 46 F>B s g-R N A i$ animals (Fig. 9G). The average length of the VNC in the control $\left(m y s^{11} /+\right)$ larvae was $794.1 \pm 106.9 \mu \mathrm{m}(n=30$ larvae $)$ compared with $648.7 \pm 93.13 \mu \mathrm{m}(n=21$ larvae $)$ in the $46 \mathrm{~F}>B s g-R N A i$ animals $\left(F_{(3,77)}=8.869, p<0.0001\right.$, ANOVA, Tukey post hoc test). The average VNC length in the $m y s^{11} /+; 46 F>B s g-R N A i$ larvae was rescued to $764.7 \pm 101.7 \mu \mathrm{m}(n=19$ larvae $)\left(F_{(3,77)}=8.869\right.$, $p=0.003$, ANOVA, Tukey post hoc test). We also investigated whether larval locomotion was rescued in the $m y s^{11} /+$ background. The average speed of the larvae was reduced from $4.9 \pm$ $2.3 \mathrm{~mm} / \mathrm{s}(n=30)$ in controls $(46 F>$ Dicer 2$)$ to $3.9 \pm 2.2 \mathrm{~mm} / \mathrm{s}$ $(n=28)$ in $46 F>B s g-R N A i$ animals $\left(F_{(3,139)}=5.292, p=0.027\right.$, ANOVA, Tukey post hoc test). The average speed of the $m y s^{11}$ / $+; 46 \mathrm{~F}>B s g-R N A i$ larvae was rescued to $4.4 \pm 1.5 \mathrm{~mm} / \mathrm{s}(n=47)$ $\left(F_{(3,139)}=5.292, p=0.037\right.$, ANOVA, Tukey post hoc test) (Fig. 9I). We also tested another loss-of-function mutant, $m y s^{1}$, and found that the peripheral glial compression phenotype was completely rescued in all samples ( $n=8$ larvae). This suggests that reducing the amount of integrin within the glia rescues both the perineurial glial compression and the VNC compression phenotypes, as well as the locomotor defects, and that these phenotypes arise as a result of integrin dysregulation. To determine whether the integrin-Bsg interaction was dependent on other focal adhesion complex components, we tested whether a loss-of-function mutant of Talin, rhea ${ }^{1}$, would rescue the Bsg knockdown phenotype. Talin is an actin-binding protein which associates with the focal adhesions via direct or indirect binding to integrin (Sun et al., 2016). Bsg knockdown in a heterozygous rhea ${ }^{l}$ background partially rescued the compression phenotype (Fig. $9 F, H$ ), with a 
A
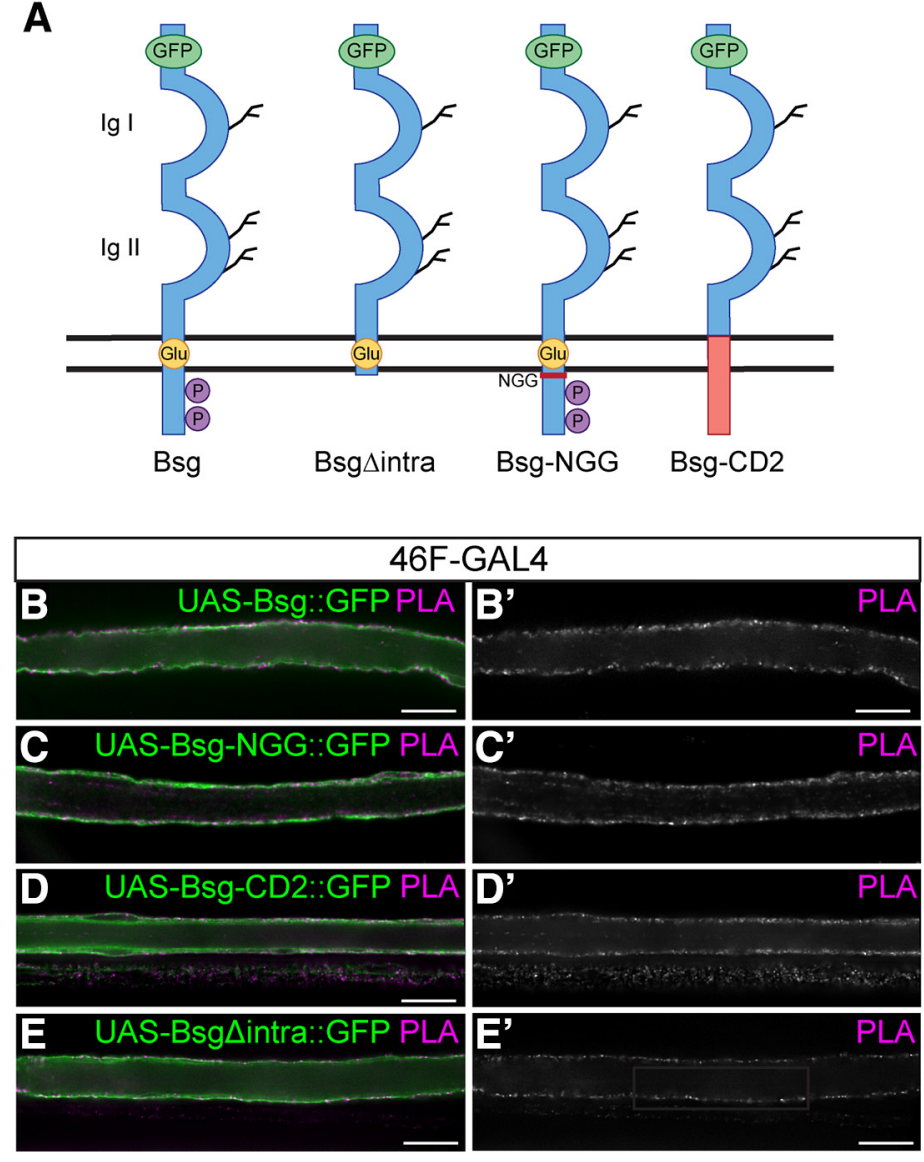

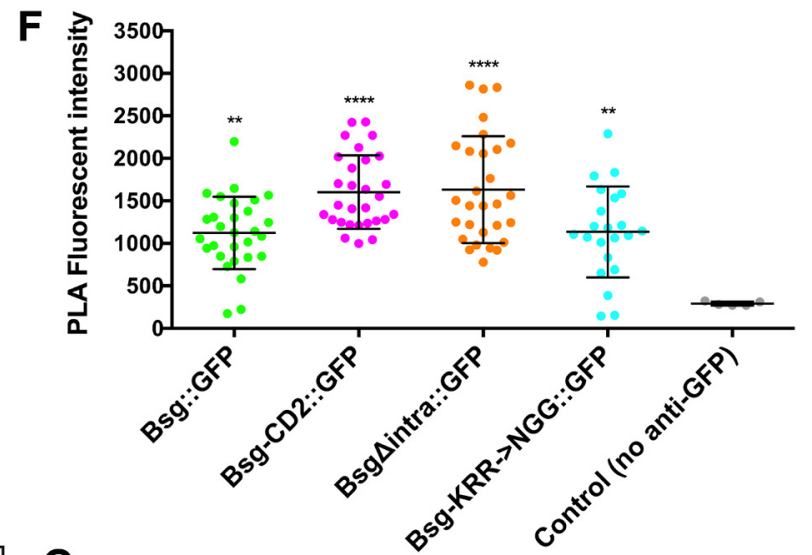

G

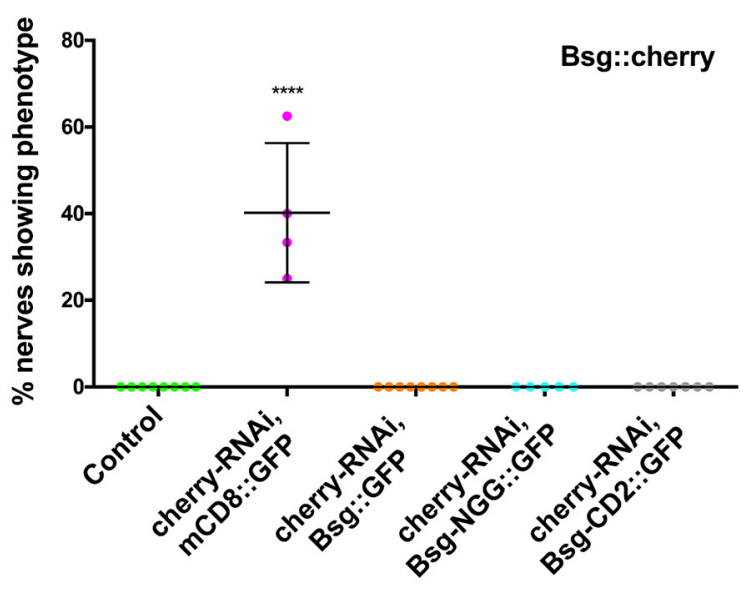

Figure 8. The extracellular domain of Bsg is sufficient for close proximity to integrins. $A$, Control and mutant Bsg constructs used in rescue experiments. Each transgene is labeled with extracellular GFP. Adapted with permission from Besse et al. (2007). B-D, PLAs testing the association of the different Bsg transgenes and the $\beta$ PS integrin (mys) subunit using the perineurial glial 46F-GAL4 driver. Scale bars, $15 \mu \mathrm{m}$. Positive PLA (magenta) was observed between Bsg::GFP and integrin in nerves expressing: UAS-Bsg::GFP (B); UAS-Bsg-NGG::GFP where conserved transmembrane residues KRR have been mutated to NGG (C); UAS-Bsg-CD2::GFP where the transmembrane and intracellular domains of Bsg have been replaced by those of rat CD2 (D); and UAS-BsgDintra::GFP construct where Bsg is lacking its intracellular domain $(\boldsymbol{E})$. $\boldsymbol{F}$, Fluorescence of PLA reactions in all UASBsg::GFP constructs was significantly higher than in control lacking anti-GFP antibody, indicating that mutations to transmembrane and intracellular domains of Bsg do not affect the Bsg-integrin interaction in the perineurial glia $\left.{ }^{* *} p<0.01,{ }^{* * * *} p<0.0001\right)$. G, Rescue of the Bsg knockdown phenotype using Bsg constructs. Knockdown of Bsg::cherry in the perineurial glia using 46F-GAL4 driving expression of cherry-RNAi and UAS-mCD8::GFP resulted in glial compression in $40.2 \%$ of nerves. This phenotype was completely rescued by coexpression of normal and mutant UAS-Bsg::GFP constructs $\left({ }^{* * *} p<0.0001\right) .{ }^{* *} p<0.01,{ }^{* * * *} p<0.0001$.

significant reduction in the penetrance of glial compression $\left(F_{(3,56)}=91.92, p<0.0001\right.$, ANOVA, Tukey post hoc test $)(n=8$ larvae). The VNC length in the $46 \mathrm{~F}>\mathrm{Bsg}_{\mathrm{B}} \mathrm{RNA}$ i; rhea $^{1} /+$ was also increased compared with the $46 \mathrm{~F}>B s g-R N A i$ animals, with an average length of $737.7 \pm 101.5 \mu \mathrm{m}$ ( $n=11$ larvae) (Fig. $9 G)$, but this change was not statistically significant $\left(F_{(3,77)}=8.869\right.$, $p=0.095$, ANOVA, Tukey post hoc test). Together, these results indicate that Bsg is interacting with integrin to maintain glial morphology, and this is mediated in part by integrin-based focal adhesion complexes.

\section{Discussion}

Previous research on Bsg has focused on its role in cancer metastasis leaving the normal biological functions of Bsg underappreciated (Xiong et al., 2014). Bsg is expressed in the vertebrate glia where it is associated with photoreceptor function as well as glioma progression (Philp et al., 2003; Miranda-Gonçalves et al., 2013; Tian et al., 2013). We found that Bsg is coexpressed with $\beta$-integrin in the perineurial glia: the cells that form the outermost glial layer surrounding the Drosophila nervous system, analogous to perineurial cells in vertebrates (Clark et al., 2014).
Bsg knockdown caused significant shortening of the VNC, as well as apparent constriction of the glia and ECM ensheathing the peripheral nerves. The effects of Bsg knockdown were rescued by loss-of-function $\beta$-integrin and Talin mutants. These findings indicate that Bsg interacts with the focal adhesions, likely serving as a negative regulator of integrin to maintain glial morphology.

We found that knockdown of Bsg in the perineurial glia caused an apparent constriction of the glial membrane in the peripheral nerves, affecting the overlying ECM and the underlying septate junctions. Although the glia and ECM appeared to be mechanically compressed, we hypothesized that the convolutions could also be a result of glial overproliferation. However, phospho-Histone3 staining for mitotic cells showed no difference from controls (data not shown). The glial phenotype also resembles the waves of membrane-cortex infolding seen in migrating cells as a result of oscillating compression and dilation (Kapustina et al., 2013), suggesting that the glia-ECM phenotype is a result of membrane compression. The phenotype in the peripheral nerves was limited to the NER of the abdominal nerves A3-A8 and was distributed randomly within the NER. During larval locomotion, the nerves are subject to significant stretching 

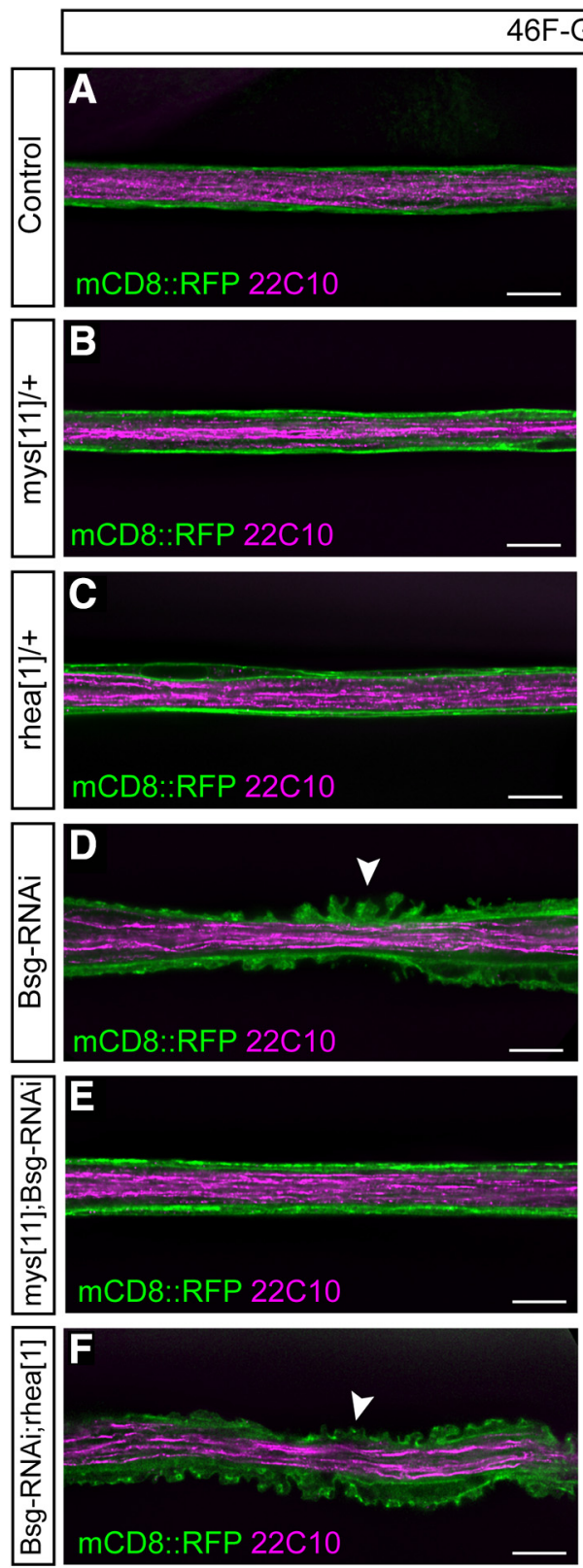
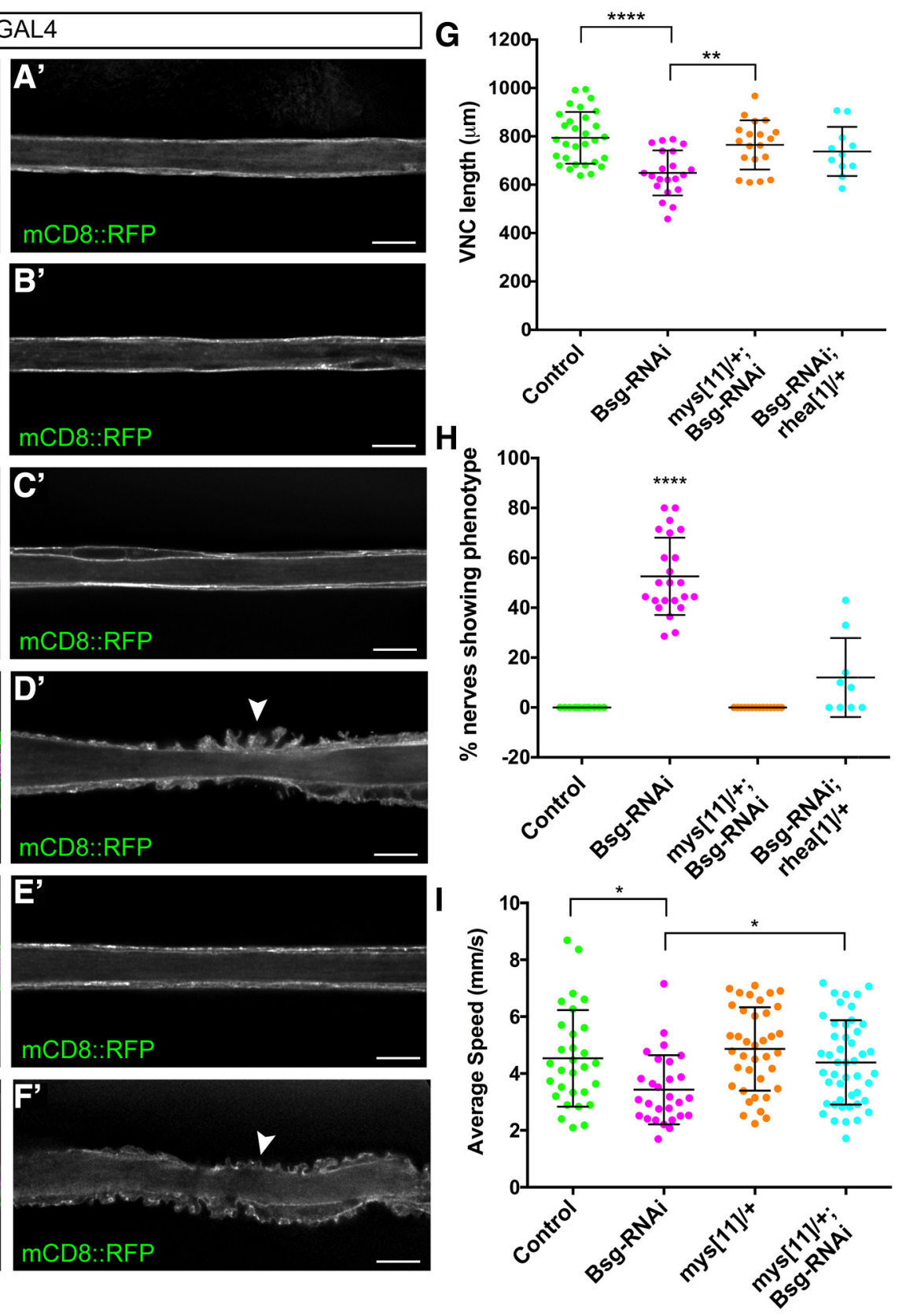

Figure 9. Reduction of focal adhesion components rescues the Bsg knockdown phenotypes. $\boldsymbol{A}$, Peripheral nerve of control (46F $>$ Dicer2) third instar larvae. Perineurial glia are labeled using 46F-GAL4 driving expression of mCD8::RFP (green), and axons are labeled using anti-22C10/Futsch (magenta). B, C, Peripheral nerve of third instar larvae with heterozygous loss-of-function mutations in $\beta$-integrin (mys ${ }^{11}, \boldsymbol{B}$ ) and Talin (rhea ${ }^{1}, \boldsymbol{C}$ shows normal glial morphology. $\boldsymbol{D}$, Knockdown of Bsg in the perineurial glia results in compression of the glial cells (arrowheads). $\boldsymbol{E}$, Knockdown of Bsg in the perineurial glia in the background of heterozygous mys ${ }^{17}$ mutant rescues the glial compression phenotype. $\boldsymbol{F}$, Knockdown of Bsg in the perineurial glia in the background of heterozygous rhea mutant partially rescues the glial phenotype, but some compressions remain (arrowhead). Scale bars, $15 \mu \mathrm{m}$. $G$, Length of VNC in perineurial glial Bsg knockdown $\left(46 \mathrm{~F}>\right.$ Bsg-RNAi) animals is significantly shorter than in control $\left(\right.$ mys $^{11} /+$ or $r$ hea $\left.{ }^{1} /+\right)$ animals $\left(^{* * * *} p>0.0001\right)$. The VNC compression was rescued in mys ${ }^{11} ; 46 F>B s g-R N A i$ animals $\left({ }^{* *} p=0.003\right)$ but not in the $46 \mathrm{~F}>B_{s g}-R N A i ;$ r rhea ${ }^{7}$ animals. $\boldsymbol{H}$, Knockdown of Bsg in the perineurial glia in the background of integrin mutant mys ${ }^{11}$ completely rescues peripheral glial compression, whereas the Talin mutant rhea ${ }^{1}$ significantly reduces the penetrance $\left(^{* * * *} p<0.0001\right)$. I, Knockdown of Bsg in the perineurial glia resulted in a significant reduction of larval locomotion, which was rescued by the heterozygous mys ${ }^{11}$ mutant $\left({ }^{*} p=0.0017\right) .{ }^{*} p<0.05,{ }^{* *} p<0.01,{ }^{* * *} p<0.0001$.

and compressing forces as the body wall muscles contract in peristaltic waves (Sun et al., 1999; Vaadia et al., 2019). We hypothesized that the cell-cell junctions between neighboring glial cells may be a "weak" spot, which would be more vulnerable to disruption, but the distribution of the phenotype suggests instead that the glial sheath is equally susceptible to stress along the nerve. The lack of a phenotype in the MFA, and in the shorter abdominal nerves A1-A2, may be because the nerves are tethered more tightly to the muscles they innervate and therefore less susceptible to mechanical stressors. The perineurial glia-ECM sheath is necessary to support peripheral nerve function and integrity, and facilitate locomotion (Petley-Ragan et al., 2016). Thus, the glial compressions and compression of the VNC may reflect both a morphologic and functional disruption. Along these lines, we observed a decrease in speed of larval locomotion when Bsg was knocked down in the perineurial glia, indicating that glial compression in the VNC and peripheral nerves affects overall function of the nervous system. 
Control

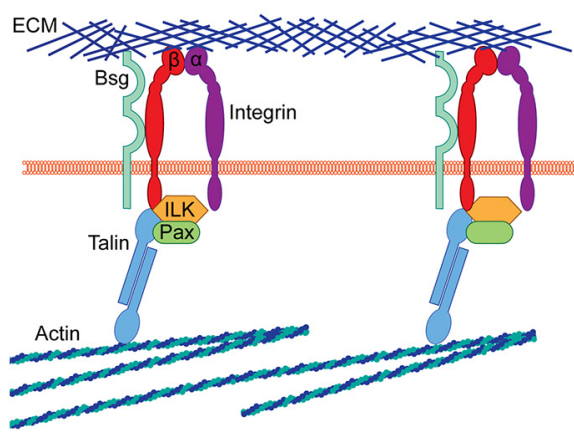

Bsg-RNAi

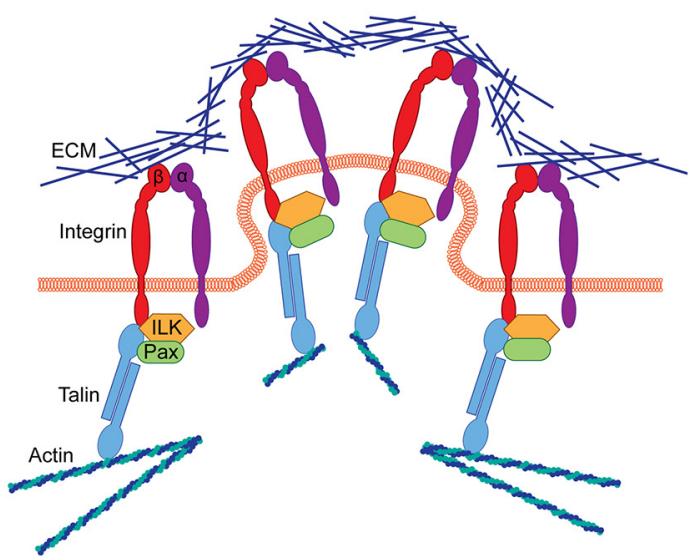

Figure 10. Model of increased integrin activation in Bsg knockdown in the perineurial glia. In controls, Bsg associates with the $\beta$-subunit of the integrin heterodimer, which binds the ECM via the head domain and the intracellular focal adhesion components, including Integrin Linked Kinase (ILK), Paxillin (Pax), and Talin, via its intracellular tail. The focal adhesion also associates with the glial actin cytoskeleton. In Bsg knockdown, there is an increase in integrin activation, which results in excessive adhesion between the ECM and the glial membrane and physical deformation of the membrane and ECM. This excess adhesion also results in breakage of the glial actin filaments under stress. It is also possible that Bsg interacts with integrin through an intermediate protein, but we favor a model of direct interaction.

We found that the constriction of the glial cells affects the integrity of the glial cytoskeleton. In regions of glial constriction, there are breakages in the actin cytoskeleton, and fragments of actin filaments appear to localize to the tips of the constricted regions. Similarly, there were overlapping gaps in the myosin motors at these regions, and the glial microtubules appear to be completely ruptured. Computer-generated simulations of actin filaments under mechanical stress have shown that compressive forces applied to actin cause the bonds between filament subunits to rupture, and that bending at a curvature of $\sim 300 \mathrm{~nm}$ will cause filament breakage (Murrell and Gardel, 2012; De La Cruz et al., 2015). Similarly, microfluidic analysis of microtubules show that they soften under mechanical stress and become more susceptible to breakage (Schaedel et al., 2015). Therefore, it is likely that glial constriction confers mechanical stress on the cytoskeleton, which causes rupturing of the cytoskeletal components. This effect could be mediated through the integrin-based focal adhesion complex. For instance, preventing Bsg-integrin interactions in hepatoma cells blocks the focal adhesion kinase pathway, resulting in rearrangement of the cellular cytoskeleton (Y. Li et al., 2012). Thus, the effects of Bsg loss could be mediated through changes to focal adhesion complex signaling.

The phenotypes we observed in glial Bsg knockdown, namely, a shortening of the VNC and the constriction of the peripheral glia and ECM, are the converse of what is seen in glial integrin knockdown. Knockdown of $\beta$-integrin in the perineurial glia prevents the adhesion between the glia and ECM necessary for ensheathment of the nerve, and results in an incomplete glial sheath (Xie and Auld, 2011). Furthermore, degradation of the ECM or knockdown of $\beta$-integrin in all glial cells causes significant lengthening of the VNC, suggesting that the glia-ECM interaction is necessary to shape the entire nervous system (Xie and Auld, 2011; Meyer et al., 2014). Together, these results suggest that Bsg plays a role in facilitating the integrity and function of the glia-ECM sheath.

We favor a model where Bsg function is mediated through interactions with integrins in the perineurial glia such that, in the absence of Bsg, integrin is dysregulated causing the glial cells to become excessively adherent to the ECM, leading to constriction of the glial-ECM sheath (Fig. 10). In Drosophila, Bsg interacts genetically and colocalizes with integrin (Reed et al., 2004; Curtin et al., 2005), and our PLA results demonstrate that Bsg and $\beta$-integrin are in close proximity in the perineurial glia. We found that reducing the gene dosage of $\beta$-integrin or Talin by $50 \%$ was sufficient to rescue the Bsg knockdown phenotype. Although we have evidence for an interaction between Bsg and $\beta$-integrin in the glia, the exact function and mechanisms underlying this interaction are still unknown. Our PLA results show that Bsg and integrin are coexpressed at the glial membrane, but it is unknown whether they directly bind in Drosophila glia. Bsg and $\beta$-integrin bind in hepatoma cells via the membrane-proximal immunoglobulin domain of Bsg and the metal ion-dependent adhesion site motif of $\beta$-integrin (Y. Li et al., 2012). Bsg and $\beta$-integrin are also clustered together on the surface of human retinal cells mediated by the cell-surface protein galectin-3 (Priglinger et al., 2013). Thus, it is possible that Bsg and integrins directly interact in the perineurial glia and that the loss of Bsg leads to a change in glial-ECM interactions. However, we cannot rule out cell-nonautonomous actions of integrin coming from the subperineurial glia, the only cell type that contacts the perineurial glia, as integrin signaling has been shown to have nonautonomous effects through adjacent cells (Long et al., 2016; Scully et al., 2016). Previous research has shown that integrins are expressed in the subperineurial glia, but their function in these cells has not been fully elucidated (Xie and Auld, 2011)

We hypothesize that, in the absence of Bsg, integrin activation is increased, leading to a subsequent increase in membrane-ECM adhesion. Increasing integrin activation leads to changes in cellECM adhesion: in cultured cells, activation of $\beta 1$-integrin increases cell traction forces, which results in spreading of cells along the culture matrix (Lin et al., 2013). Overexpression of integrins at the cell membrane causes an increase in cell-matrix adhesion in vitro (Nakada et al., 2013; Hayashido et al., 2014). How the absence of Bsg might lead to integrin activation is still an open question and could occur through intracellular or extracellular mechanisms. Extracellularly, ECM proteins cause integrin dimers to cluster and become stabilized in their high-affinity ("open") state, recruiting intracellular effectors to facilitate downstream signaling ( $\mathrm{Hu}$ and Luo, 2013). The function of Bsg may be 
to attenuate this activation through an extracellular interaction, as we found that expression of the extracellular domain of Bsg was sufficient to rescue the knockdown phenotype. These data suggest that the presence of the extracellular domain of Bsg prevents excess activation of integrin via an interaction with the extracellular head of $\beta$-integrin. Intracellularly, binding of adaptor proteins, such as Talin, to the integrin tail can also induce conformational changes, resulting in integrin activation (Calderwood, 2004). However, we find that this model is less likely to fit our data due to the fact that a $50 \%$ decrease in the gene dose of Talin did not fully rescue the phenotype. Given that loss of Talin or blocking Talin-integrin binding reduces integrin activation (Tadokoro et al., 2003; Tanentzapf and Brown, 2006), it is likely that reduction in Talin can partially rescue the Bsg knockdown phenotype due to a reduction in integrin activation. It is also possible that Bsg is acting on integrin through an intermediate factor, as Bsg is highly pleiotropic with a wide variety of binding partners (Muramatsu, 2016), which would make it difficult to identify potential intermediate interactions.

In conclusion, we have identified a role for Bsg in the maintenance of the glia-ECM sheath of the Drosophila nervous system. Bsg is expressed at the glial membrane where it associates extracellularly with integrin, and via negative regulation of integrins in the focal adhesion complexes upholds the structure of the gliaECM sheath. Further studies into the interaction between Bsg and the focal adhesions may reveal the exact mechanism of the Bsg-integrin interaction.

\section{References}

Barros LF, Kanaseki T, Sabirov R, Morishima S, Castro J, Bittner CX, Maeno E, Ando-Akatsuka Y, Okada Y (2003) Apoptotic and necrotic blebs in epithelial cells display similar neck diameters but different kinase dependency. Cell Death Differ 10:687-697.

Bateman JR, Lee AM, Wu CT (2006) Site-specific transformation of Drosophila via $\phi \mathrm{C} 31$ integrase-mediated cassette exchange. Genetics 173:769-777.

Berditchevski F, Chang S, Bodorova J, Hemler ME (1997) Generation of monoclonal antibodies to integrin-associated proteins: evidence that $\alpha 3 \beta 1$ complexes with EMMPRIN/Basigin/OX47/M6. J Biol Chem 272:29174-29180.

Besse F, Mertel S, Kittel RJ, Wichmann C, Rasse TM, Sigrist SJ, Ephrussi A (2007) The Ig cell adhesion molecule Basigin controls compartmentalization and vesicle release at Drosophila melanogaster synapses. J Cell Biol 177:843-855.

Brower DL, Wilcox M, Piovant M, Smith RJ, Reger LA (1984) Related cellsurface antigens expressed with positional specificity in Drosophila imaginal discs. Proc Natl Acad Sci USA 81:7485-7489.

Brown NH, Gregory SL, Rickoll WL, Fessler LI, Prout M, White RA, Fristrom JW (2002) Talin is essential for integrin function in Drosophila. Dev Cell 3:569-579.

Calderwood DA (2004) Talin controls integrin activation. Biochem Soc Trans 32:434-437.

Calderwood DA, Zent R, Grant R, Rees DJ, Hynes RO, Ginsberg MH (1999) The Talin head domain binds to integrin beta subunit cytoplasmic tails and regulates integrin activation. J Biol Chem 274:28071-28074.

Clark JK, Ashley O, Mastracci TL, Sussel L, Matise MP, Kucenas S (2014) Mammalian Nkx2.2 perineurial glia are essential for motor nerve development. Dev Dyn 243:1116-1129.

Colognato H, Tzvetanova ID (2011) Glia unglued: how signals from the extracellular matrix regulate the development of myelinating glia. Dev Neurobiol 71:924-955.

Curtin KD, Meinertzhagen IA, Wyman RJ (2005) Basigin (EMMPRIN/ CD147) interacts with integrin to affect cellular architecture. J Cell Sci 118:2649-2660.

Curtin KD, Wyman RJ, Meinertzhagen IA (2007) Basigin/EMMPRIN/ CD147 mediates neuron-glia interactions in the optic lamina of Drosophila. Glia 55:1542-1425.
De La Cruz EM, Martiel JL, Blanchoin L (2015) Mechanical heterogeneity favors fragmentation of strained actin filaments. Biophys J 108:22702281.

Dietzl G, Chen D, Schnorrer F, Su KC, Barinova Y, Fellner M, Gasser B, Kinsey K, Oppel S, Scheiblauer S, Couto A, Marra V, Keleman K, Dickson BJ (2007) A genome-wide transgenic RNAi library for conditional gene inactivation in Drosophila. Nature 448:151-156.

Feltri ML, Porta DG, Previtali SC, Nodari A, Migliavacca B, Cassetti A, Littlewood-Evans A, Reichardt LF, Messing A, Quattrini A, Mueller U, Wrabetz L (2002) Conditional disruption of $\beta 1$ integrin in Schwann cells impedes interactions with axons. J Cell Biol 156:199-210.

Hatan M, Shinder V, Israeli D, Schnorrer F, Volk T (2011) The Drosophila blood brain barrier is maintained by GPCR-dependent dynamic actin structures. J Cell Biol 192:307-319.

Hayashido Y, Kitano H, Sakaue T, Fujii T, Suematsu M, Sakurai S, Okamoto $\mathrm{T}$ (2014) Overexpression of integrin $\alpha \mathrm{v}$ facilitates proliferation and invasion of oral squamous cell carcinoma cells via MEK/ERK signaling pathway that is activated by interaction of integrin $\alpha \mathrm{v} \beta 8$ with type I collagen. Int J Oncol 45:1875-1882.

$\mathrm{Hu}$ P, Luo BH (2013) Integrin bi-directional signaling across the plasma membrane. J Cell Physiol 228:306-312.

Janke C, Montagnac G (2017) Causes and consequences of microtubule acetylation. Curr Biol 27:R1287-R1292.

Kapustina M, Elston TC, Jacobson K (2013) Compression and dilation of the membrane-cortex layer generates rapid changes in cell shape. J Cell Biol 200:95-108.

Kaushik DK, Hahn JN, Yong VW (2015) EMMPRIN, an upstream regulator of MMPs, in CNS biology. Matrix Biol 44:138-146.

Kirk P, Wilson MC, Heddle C, Brown MH, Barclay AN, Halestrap AP (2000) CD147 is tightly associated with lactate transporters MCT1 and MCT4 and facilitates their cell surface expression. EMBO J 19:3896-3904.

Lee T, Luo L (1999) Mosaic analysis with a repressible cell marker for studies of gene function in neuronal morphogenesis. Neuron 22:451-461.

Li H, Berlin Y, Hart RP, Grumet M (2003) Microtubules are critical for radial glial morphology: possible regulation by MAPs and MARKs. Glia 44:3746.

Li J, Su Y, Xia W, Qin Y, Humphries MJ, Vestweber D, Cabañas C, Lu C, Springer TA (2017) Conformational equilibria and intrinsic affinities define integrin activation. EMBO J 36:629-645.

Li Y, Wu J, Song F, Tang J, Wang SJ, Yu XL, Chen ZN, Jiang JL (2012) Extracellular membrane-proximal domain of HAb18G/CD147 binds to metal ion-dependent adhesion site (MIDAS) motif of integrin $\beta 1$ to modulate malignant properties of hepatoma cells. J Biol Chem 287:47594772.

Lin GL, Cohen DM, Desai RA, Breckenridge MT, Gao L, Humphries MJ, Chen CS (2013) Activation of beta 1 but not beta 3 integrin increases cell traction forces. FEBS Lett 587:763-769.

Liu L, MacKenzie KR, Putluri N, Maletić-Savatić M, Bellen HJ (2017) The glia-neuron lactate shuttle and elevated ROS promote lipid synthesis in neurons and lipid droplet accumulation in glia via APOE/D. Cell Metab 26:1-19.

Long K, Moss L, Laursen L, Boulter L, Ffrench-Constant C (2016) Integrin signalling regulates the expansion of neuroepithelial progenitors and neurogenesis via Wnt7a and Decorin. Nat Commun 7:10354.

Manoharan C, Wilson MC, Sessions RB, Halestrap AP (2006) The role of charged residues in the transmembrane helices of monocarboxylate transporter 1 and its ancillary protein Basigin in determining plasma membrane expression and catalytic activity. Mol Membr Biol 23:486498.

Meyer S, Schmidt I, Klämbt C (2014) Glia ECM interactions are required to shape the Drosophila nervous system. Mech Dev 133:105-116.

Miranda-Gonçalves V, Honavar M, Pinheiro C, Martinho O, Pires MM, Pinheiro C, Cordeiro M, Bebiano G, Costa P, Palmeirim I, Reis RM, Baltazar F (2013) Monocarboxylate transporters (MCTs) in gliomas: expression and exploitation as therapeutic targets. Neuro Oncol 15:172188.

Morin X, Daneman R, Zavortink M, Chia W (2001) A protein trap strategy to detect GFP-tagged proteins expressed from their endogenous loci in Drosophila. Proc Natl Acad Sci USA 98:15050-15055.

Muramatsu T (2016) Basigin (CD147), a multifunctional transmembrane glycoprotein with various binding partners. J Biochem 159:481-490. 
Murrell MP, Gardel ML (2012) F-actin buckling coordinates contractility and severing in a biomimetic actomyosin cortex. Proc Natl Acad Sci USA 109:20820-20825.

Nagarkar-Jaiswal S, Lee PT, Campbell ME, Chen K, Anguiano-Zarate S, Gutierrez MC, Busby T, Lin WW, He Y, Schulze KL, Booth BW, EvansHolm M, Venken KJ, Levis RW, Spradling AC, Hoskins RA, Bellen HJ (2015) A library of MiMICs allows tagging of genes and reversible, spatial and temporal knockdown of proteins in Drosophila. Elife 2015:4.

Nakada M, Nambu E, Furuyama N, Yoshida Y, Takino T, Hayashi Y, Sato H, Sai Y, Tsuji T, Miyamoto K, Hirao A, Hamada J (2013) Integrin $\alpha 3$ is overexpressed in glioma stem-like cells and promotes invasion. $\mathrm{Br} \mathrm{J}$ Cancer 108:2516-2524.

Nussbaum-Krammer CI, Neto MF, Brielmann RM, Pedersen JS, Morimoto RI (2015) Investigating the spreading and toxicity of prion-like proteins using the metazoan model organism C. elegans. J Vis Exp 95:52321.

O'Toole TE, Mandelman D, Forsyth J, Shattil SJ, Plow EF, Ginsberg MH, Ginsberg MH, Calderwood DA (1991) Modulation of the affinity of integrin alpha IIb beta 3 (GPIIb-IIIa) by the cytoplasmic domain of alpha IIb. Science 254:845-847.

Ozeki N, Kawai R, Hase N, Hiyama T, Yamaguchi H, Kondo A, Nakata K, Mogi M (2015) $\alpha 2$ integrin, extracellular matrix metalloproteinase inducer, and matrix metalloproteinase-3 act sequentially to induce differentiation of mouse embryonic stem cells into odontoblast-like cells. Exp Cell Res 331:21-37.

Pastor-Pareja JC, Xu T (2011) Shaping cells and organs in Drosophila by opposing roles of fat body-secreted collagen IV and Perlecan. Dev Cell 21:245-256

Petley-Ragan LM, Ardiel EL, Rankin CH, Auld VJ (2016) Accumulation of laminin monomers in Drosophila glia leads to glial endoplasmic reticulum stress and disrupted larval locomotion. J Neurosci 36:1151-1164.

Philp NJ, Ochrietor JD, Rudoy C, Muramatsu T, Linser PJ (2003) Loss of MCT1, MCT3, and MCT4 expression in the retinal pigment epithelium and neural retina of the 5A11/Basigin-null mouse. Invest Ophthalmol Vis Sci 44:1305-1311.

Potokar M, Kreft M, Li L, Andersson DJ, Pangršič T, Chowdhury HH, Pekny M, Zorec R (2007) Cytoskeleton and vesicle mobility in astrocytes. Traffic $8: 12-20$.

Preibisch S, Saalfeld S, Tomancak P (2009) Globally optimal stitching of tiled 3D microscopic image acquisitions. Bioinformatics 25:1463-1465.

Priglinger CS, Szober CM, Priglinger SG, Merl J, Euler KN, Kernt M, Gondi G, Behler J, Geerlof A, Kampik A, Ueffing M, Hauck SM (2013) Galectin3 induces clustering of CD147 and integrin- $\beta 1$ transmembrane glycoprotein receptors on the RPE cell surface. PLoS One 8:e70011.

Reed BH, Wilk R, Schöck F, Lipshitz HD (2004) Integrin-dependent apposition of Drosophila extraembryonic membranes promotes morphogenesis and prevents anoikis. Curr Biol 14:372-380.

Riedl J, Crevenna AH, Kessenbrock K, Yu JH, Neukirchen D, Bista M, Bradke F, Jenne D, Holak TA, Werb Z, Sixt M, Wedlich-Soldner R (2008) Lifeact: a versatile marker to visualize F-actin. Nat Methods 5:605-607.

Royer LA, Weigert M, Günther U, Maghelli N, Jug F, Sbalzarini IF, Myers EW (2015) ClearVolume: open-source live 3D visualization for lightsheet microscopy. Nat Methods 12:480-481.

Samarasekera G, Auld VJ (2018) C-terminal Src kinase (Csk) regulates the tricellular junction protein Gliotactin independent of Src. Mol Biol Cell 29:123-136

Schaedel L, John K, Gaillard J, Nachury MV, Blanchoin L, Théry M (2015) Microtubules self-repair in response to mechanical stress. Nat Mater 14:1156-1163.

Schindelin J, Arganda-Carreras I, Frise E, Kaynig V, Longair M, Pietzsch T, Preibisch S, Rueden C, Saalfeld S, Schmid B, Tinevez JY, White DJ, Hartenstein V, Eliceiri K, Tomancak P, Cardona A (2012) Fiji: an opensource platform for biological-image analysis. Nat Methods 9:676-682.
Schneider CA, Rasband WS, Eliceiri KW (2012) NIH Image to ImageJ: 25 years of image analysis. Nat Methods 9:671-675.

Scully KM, Skowronska-Krawczyk D, Krawczyk M, Merkurjev D, Taylor H, Livolsi A, Tollkuhn J, Stan RV, Rosenfeld MG (2016) Epithelial cell integrin $\beta 1$ is required for developmental angiogenesis in the pituitary gland. Proc Natl Acad Sci USA 113:13408-13413.

Sepp KJ, Auld VJ (1999) Conversion of lacZ enhancer trap lines to GAL4 lines using targeted transposition in Drosophila melanogaster. Genetics 151:1093-1101

Sepp KJ, Schulte J, Auld VJ (2000) Developmental dynamics of peripheral glia in Drosophila melanogaster. Glia 30:122-133.

Sepp KJ, Schulte J, Auld VJ (2001) Peripheral glia direct axon guidance across the CNS/PNS transition zone. Dev Biol 238:47-63.

Söderberg O, Gullberg M, Jarvius M, Ridderstråle K, Leuchowius KJ, Jarvius J, Wester K, Hydbring P, Bahram F, Larsson LG, Landegren U (2006) Direct observation of individual endogenous protein complexes in situ by proximity ligation. Nat Methods 3:995-1000.

Sun B, Xu P, Salvaterra PM (1999) Dynamic visualization of nervous system in live Drosophila. Proc Natl Acad Sci USA 96:10438-10443.

Sun Z, Guo SS, Fässler R (2016) Integrin-mediated mechanotransduction. J Cell Biol 215:445-456.

Swierczek NA, Giles AC, Rankin CH, Kerr RA (2011) High-throughput behavioral analysis in C. elegans. Nat Methods 8:592-598.

Tadokoro S, Shattil S, Eto K, Tai V, Liddington R, de Pereda J, Ginsberg M, Calderwood D (2003) Talin binding to integrin $\beta$ tails: a final common step in integrin activation. Science 302:103-106.

Tanentzapf G, Brown NH (2006) An interaction between integrin and the talin FERM domain mediates integrin activation but not linkage to the cytoskeleton. Nat Cell Biol 8:601-606.

Tian L, Zhang Y, Chen Y, Cai M, Dong H, Xiong L (2013) EMMPRIN is an independent negative prognostic factor for patients with astrocytic glioma. PLoS One 8:e58069.

Vaadia RD, Li W, Voleti V, Singhania A, Hillman EM, Grueber WB (2019) Characterization of proprioceptive system dynamics in behaving Drosophila larvae using high-speed volumetric microscopy. Curr Biol 29:935-944.e4.

Velentzas PD, Zhang L, Das G, Chang TK, Nelson C, Kobertz WR, Baehrecke EH (2018) The proton-coupled monocarboxylate transporter Hermes is necessary for autophagy during cell death. Dev Cell 47:281293.e4.

Venken KJ, Schulze KL, Haelterman NA, Pan H, He Y, Evans-Holm M, Carlson JW, Levis RW, Spradling AC, Hoskins RA, Bellen HJ (2011) MiMIC: a highly versatile transposon insertion resource for engineering Drosophila melanogaster genes. Nat Methods 8:737-743.

Wang S, Yoo S, Kim H, Wang M, Zheng C, Parkhouse W, Krieger C, Harden $\mathrm{N}$ (2015) Detection of in situ protein-protein complexes at the Drosophila larval neuromuscular junction using proximity ligation assay. J Vis $\operatorname{Exp}$ 95:52139.

Wieschaus E, Nüsslein-Volhard C, Jürgens G (1984) Mutations affecting the pattern of the larval cuticle in Drosophila melanogaster Roux's. Wilehm Roux Arch Dev Biol 193:296-307.

Wilson MC, Meredith D, Halestrap AP (2002) Fluorescence resonance energy transfer studies on the interaction between the lactate transporter MCT1 and CD147 provide information on the topology and stoichiometry of the complex in situ. J Biol Chem 277:3666-3672.

Wright TR (1960) The phenogenetics of the embryonic mutant lethal myospheroid, in Drosophila melanogaster. J Exp Zool 143:77-99.

Xie X, Auld VJ (2011) Integrins are necessary for the development and maintenance of the glial layers in the Drosophila peripheral nerve. Development 138:3813-3822.

Xiong L, Edwards C, Zhou L (2014) The biological function and clinical utilization of CD147 in human diseases: a review of the current scientific literature. Int J Mol Sci 15:17411-17441. 